\title{
Nucleosynthetic history of elements in the Galactic disk $[\mathrm{X} / \mathrm{Fe}]$-age relations from high-precision spectroscopy ${ }^{\star}$
}

\author{
L. Spina ${ }^{1}$, J. Meléndez ${ }^{1}$, A. I. Karakas² ${ }^{2}$ I. Ramírez ${ }^{3}$, T. R. Monroe ${ }^{4}$, M. Asplund ${ }^{5}$, and D. Yong ${ }^{5}$ \\ 1 Universidade de São Paulo, IAG, Departamento de Astronomia, Rua do Matão 1226, 05509-900 São Paulo, Brasil \\ e-mail: lspina@usp.br \\ 2 Monash Centre for Astrophysics, School of Physics and Astronomy, Monash University, 3800 Victoria, Australia \\ 3 Department of Astronomy, University of Texas at Austin, 2515 Speedway, Stop C1400, Austin, TX 78712-1205, USA \\ ${ }^{4}$ Space Telescope Science Institute, 3700 San Martin Drive, Baltimore, MD 21218, USA \\ 5 Research School of Astronomy and Astrophysics, The Australian National University, Cotter Road, 2611 Canberra, Australia
}

Received 18 March 2016 / Accepted 15 June 2016

\begin{abstract}
Context. The chemical composition of stars is intimately linked to the formation and evolution of the Galaxy.

Aims. We aim to trace the chemical evolution of the Galactic disk through the inspection of the [X/Fe]-age relations of 24 species from $\mathrm{C}$ to $\mathrm{Eu}$.

Methods. Using high-resolution and high signal-to-noise UVES spectra of nine solar twins, we obtained precise estimates of stellar ages and chemical abundances. These determinations have been integrated with additional accurate age and abundance determinations from recent spectroscopic studies of solar twins existing in the literature, comprising superb abundances with 0.01 dex precision. Based on this data set, we outlined the $[\mathrm{X} / \mathrm{Fe}]-$ age relations over a time interval of $10 \mathrm{Gyr}$.

Results. We present the $[\mathrm{X} / \mathrm{Fe}]$ - age relations for 24 elements $(\mathrm{C}, \mathrm{O}, \mathrm{Na}, \mathrm{Mg}, \mathrm{Al}, \mathrm{Si}, \mathrm{S}, \mathrm{K}, \mathrm{Ca}, \mathrm{Sc}, \mathrm{Ti}, \mathrm{V}, \mathrm{Cr}, \mathrm{Mn}, \mathrm{Co}, \mathrm{Ni}, \mathrm{Cu}$, $\mathrm{Zn}, \mathrm{Y}, \mathrm{Ba}, \mathrm{La}, \mathrm{Ce}, \mathrm{Nd}$, and $\mathrm{Eu}$ ). Each different class of elements showed a distinct evolution with time that relies on the different characteristics, rates, and timescales of the nucleosynthesis sites from which they are produced. The $\alpha$-elements are characterized by a $[\mathrm{X} / \mathrm{Fe}]$ decrease with time. Strikingly, the opposite behavior is observed for Ca. The iron-peak elements show an early $[\mathrm{X} / \mathrm{Fe}]$ increase followed by a decrease towards the youngest stars. The $[\mathrm{X} / \mathrm{Fe}]$ for the $n$-capture elements decrease with age. We also found that both $[\mathrm{Mg} / \mathrm{Y}]$ and $[\mathrm{Al} / \mathrm{Y}]$ are precise stellar clocks, with $[\mathrm{Al} / \mathrm{Y}]$ showing the steepest dependence on age.

Conclusions. Knowledge of the $[\mathrm{X} / \mathrm{Fe}]$-age relations is a gold mine from which we can achieve a great understanding of the processes that governed the formation and evolution of the Milky Way. Through the reverse engineering of these relations we will be able to put strong constraints on the nature of the stellar formation history, the SNe rates, the stellar yields, and the variety of the SNe progenitors.
\end{abstract}

Key words. stars: abundances - stars: fundamental parameters - stars: solar-type - Galaxy: abundances - Galaxy: disk Galaxy: evolution

\section{Introduction}

"The principal value of abundance determinations from stellar spectra is the clues they give to the nuclear history of stellar matter and, more generally, of the matter in the whole Galaxy" (Cayrel \& Cayrel de Strobel 1966). Fifty years after this pioneering work on stellar chemical abundances, the study of the chemical evolution of our Galaxy is still one of the main research areas in modern astrophysics.

Important hints regarding the formation and evolution of galaxies are locked into the chemical compositions of stars. All metals originate from stars that enrich the interstellar medium (ISM) with their own unique pattern of elements depending on their mass and initial metallicity. In fact, each specific element is produced by different sites of nucleosynthesis that contribute to the chemical evolution of galaxies with different timescales.

The Type II supernovae (SNe II) are the evolutionary termi of the most massive stars $\left(M \gtrsim 10 M_{\odot}\right)$ and are mainly responsible for the production of $\alpha$-elements (e.g., C, O, Mg, Si, S, Ca, $\mathrm{Ti})$. Since massive stars have very short lifetimes $\left(\lesssim 10^{-2} \mathrm{Gyr}\right)$, the SNe II have polluted the interstellar medium (ISM) since

* Based on observations obtained at the ESO VLT at Paranal Observatory (Observing program 083.D-0871). the very early stages of the Galaxy's evolution (see Matteucci 2014). On the other hand, the Type Ia supernovae (SNe Ia) explosions are thought to be the final fate of white dwarfs gradually accreting mass from a binary companion (single-degenerate scenario) or the result of the merger of two white dwarfs (doubledegenerate scenario); they are important producers of iron-peak elements (e.g., Cr, Mn, Co, Ni). Owing to the relatively low mass of the SNe Ia progenitors, the timescales on which they operate are typically longer $(\sim 1 \mathrm{Gyr})$ than those of SNe II. However, the SN Ia play a somewhat ambiguous role in astrophysics owing to the large variety of progenitors that are responsible for different SN Ia subclasses with diverging properties (see Hillebrandt et al. 2013 for a recent review). For instance, among the different SNe Ia, the existence of a class has been proposed that operated shortly after $(\sim 100 \mathrm{Myr})$ the beginning of the stellar formation and that could have played a role in the early Galactic evolution, but which seem to be a minor fraction $(<15-20 \%)$ of the SNe Ia (Bonaparte et al. 2013).

Last but not least, all the elements heavier than $\mathrm{Zn}(Z>$ 30) can be produced in different environments; they are commonly called neutron-capture (or $n$-capture) elements, since they are synthesized in stars through $n$-capture processes: neutrons are fused into nuclei that, if unstable, can $\beta$ decay transforming 
neutrons into protons. There are two types of $n$-capture processes: if the neutron capture timescale is longer than the decay timescale, there is a slow- $(s-)$ process, otherwise a rapid- $(r-)$ process occurs. The neutron-capture timescales are largely determined by the neutron number densities $\left(n_{\mathrm{n}}\right)$ : the $s$-process occurs when $n_{\mathrm{n}} \lesssim 10^{8} \mathrm{~cm}^{-3}$ (Busso et al. 1999), while the $r$-process takes place with much higher densities $\left(n_{\mathrm{n}} \sim 10^{24-28} \mathrm{~cm}^{-3}\right.$; Kratz et al. 2007, and references therein). This means that the two processes require very different astrophysical environments: the $s$-process is thought to occur in low- and intermediatemass stars (i.e., 1-8 $M_{\odot}$ ) during their AGB phase, while the large $n_{\mathrm{n}}$ required for the $r$-process suggests that they likely occur during SN explosions (e.g., Arlandini et al. 1999; Sneden et al. 2008; Karakas 2010; Winteler et al. 2012; Fishlock et al. 2014; Karakas \& Lattanzio 2014). However, it has also been argued that not all SN explosions can host $r$-process. Namely, only lowmass $\left(\lesssim 11 M_{\odot}\right) \mathrm{SNe}$ are likely sites for the synthesis of $r$-process elements (see Cowan \& Sneden 2004, and references therein). Nevertheless, other sites have also been hypothesized as being responsible for the production of these elements, including colliding neutron stars (Argast et al. 2004; Korobkin et al. 2012) and black hole/neutron star mergers (Surman et al. 2008); however, the actual production site(s) of the $r$-process are not known at present (Cowan \& Sneden 2004; Thielemann et al. 2011).

Owing to this variety of nucleosynthesis channels, the abundance ratios of two elemental species having different origins and characteristic timescales for release into the ISM can behave as cosmic clocks by which the formation timescales of various stellar populations (e.g., thin and thick disk) can be determined (e.g., Gilmore et al. 1989; Chiappini et al. 1997; Recio-Blanco et al. 2014; Haywood et al. 2013). Through this information it could also be possible to reveal additional clues on the stellar formation rate, the initial mass function, the mass and metallicity of the $\mathrm{SNe}$ progenitors, the occurrence of $\mathrm{SNe}$ and AGB stars, and their timescales of elemental pollution (e.g., Tinsley 1979; Matteucci \& Greggio 1986; Yoshii et al. 1996; Matteucci \& Recchi 2001; Venn et al. 2004; Pipino \& Matteucci 2009; Tsujimoto \& Shigeyama 2012; Snaith et al. 2014, 2015; Haywood et al. 2015, 2016; Jiménez et al. 2015; Vincenzo et al. 2016).

In addition, knowledge of the relations between the abundance ratios and age would put strong constraints on all these variables. However, the difficulties in providing precise age determinations of main-sequence stars and the agemetallicity degeneracy of stellar populations over recent decades have prevented us from achieving accurate information on the age-abundances dependencies (Edvardsson et al. 1993). Hints of these relations have been achieved for $\alpha$ and $n$-capture elements in field stars (Bensby et al. 2007; Haywood et al. 2013; Snaith et al. 2015; Battistini \& Bensby 2016). Additional clues come from the study of the chemical composition of open clusters for which ages are much better constrained than field stars (D'Orazi et al. 2009; Maiorca et al. 2011). These studies have revealed an increasing contribution of $s$-process elements with time from low-mass (1-4 $M_{\odot}$ ) AGB stars. Also, Magrini et al. (2009) have noted that the $[\alpha / \mathrm{Fe}]$ ratio is slightly higher for the oldest clusters. Finally, recent studies that investigated the $[\alpha / \mathrm{Fe}]$ ratios in field stars have provided interesting insights on the formation and evolution of the Galactic disk (Haywood et al. 2013, 2016; Snaith et al. 2015).

Only recently, a series of spectroscopic studies (Meléndez et al. 2012; 2014; Bedell et al. 2014; Ramírez et al. 2014a; Nissen 2015; Biazzo et al. 2015; Spina et al. 2016) have shown that, through accurate equivalent width (EW) measurements of high-quality spectra and a strict line-by-line excitation/ionization balance analysis relative to the solar spectrum, it is possible to achieve chemical abundances at sub-0.01 dex precision and extremely accurate atmospheric parameters and stellar ages.

Using this approach, Nissen (2015, hereafter N15) determined the atmospheric parameters, ages, and high-precision abundances of 14 elements from $\mathrm{C}$ to $\mathrm{Y}$ in a sample of 21 solar twins, spanning an age interval of eight Gyr. This analysis has revealed for several elements the existence of tight linear correlations between $[\mathrm{X} / \mathrm{Fe}]$ and stellar ages. These correlations are positive for most of the elements from $\mathrm{C}$ to $\mathrm{Zn}$; the only exceptions are $\mathrm{Ca}$ and $\mathrm{Cr}$, which respectively show a flat and a slightly negative correlation. In addition, $\mathrm{Y}$ decreases with increasing age, in agreement with the previous studies on $s$-process elements. On the other hand, N15 noted that the elements $\mathrm{Na}$ and $\mathrm{Ni}$ do not seem to be well correlated with the stellar ages. Recently, Spina et al. (2016, hereafter S16) analyzed a sample of 14 solar twins and they studied the $[\mathrm{X} / \mathrm{Fe}]-$ age relations for all the elements analyzed by N15 plus another eight species. They confirmed the general behavior previously found by N15, but for C, $\mathrm{O}, \mathrm{Na}, \mathrm{S}, \mathrm{Ni}$, and $\mathrm{Zn}$ they found greater $[\mathrm{X} / \mathrm{Fe}]$-age slopes compared to those found by N15. They suggested that this difference may be due to a turnover in the $[\mathrm{X} / \mathrm{Fe}]-$ age relations occurring at $\sim 6$ Gyr. In fact, while the sample studied by N15 covers ages up to $8 \mathrm{Gyr}$, all the solar twins considered by S16 are younger than $6 \mathrm{Gyr}$ and partially sample the non-linear relations (see $\mathrm{Na}$ in Fig. 1).

In the present paper we aim to provide new key insights into the $[\mathrm{X} / \mathrm{Fe}]$ vs. age relations for 24 species from $\mathrm{C}$ to $\mathrm{Eu}$, through the exploitation of high-quality data of solar twins spanning ages from 0.5 to 10 Gyr. In Sect. 2 we describe the spectral analysis and the determination of $[\mathrm{X} / \mathrm{Fe}]$ ratios and stellar ages. The $[\mathrm{X} / \mathrm{Fe}]$ vs. age relations are discussed in Sect. 3. In Sect. 4 we present our concluding remarks.

\section{Data set}

A part of the data set that we employed for our study comes from the observation and spectral analysis of a new study of nine solar twins. The resulting information has been integrated with highprecision $[\mathrm{X} / \mathrm{Fe}]$ ratios and ages for solar twins taken from the literature.

\subsection{Spectroscopic observations and data analysis}

A sample of nine solar twins were observed using UVES mounted on the Very Large Telescope during the nights 29-30 August 2009 (program 083.D-0871). The UVES spectrograph was used in the configurations that cover $306-387 \mathrm{~nm}$ in the blue and $480-1020 \mathrm{~nm}$ in the red chip. Each star was observed with a spectral resolution of $R \sim 65000$ in the blue and 110000 in the red. The final signal-to-noise ratios $(\mathrm{S} / \mathrm{N})$ are within 750 and 1300 pixel $^{-1}$ at $672 \mathrm{~nm}$ with a median of $\sim 1000$. In addition to the solar twins, a short exposure of Juno was performed to acquire a solar spectrum with a S/N of 1250 pixel $^{-1}$. The UVES data was reduced by hand using IRAF, as described by Monroe et al. (2013) and Tucci Maia et al. (2015).

Our method of analysis of the nine solar twin spectra is similar to that described by Spina et al. (2016). Namely, we employed a master list of atomic transitions from Meléndez et al. (2014) that includes 86 lines of Fe I, 14 of Fe II, and 200 lines of other elements. Using the IRAF splot task and adopting the 
Table 1. Stellar parameters.

\begin{tabular}{c|cccc|cc}
\hline \hline Star & $\begin{array}{c}T_{\text {eff }} \\
{[\mathrm{K}]}\end{array}$ & $\begin{array}{c}\log \mathrm{g} \\
{[\mathrm{dex}]}\end{array}$ & $\begin{array}{c}{[\mathrm{Fe} / \mathrm{H}]} \\
{[\mathrm{dex}]}\end{array}$ & $\begin{array}{c}\xi \\
{\left[\mathrm{km} \mathrm{s}^{-1}\right]}\end{array}$ & $\begin{array}{c}\text { Age } \\
{[\mathrm{Gyr}]}\end{array}$ & $\begin{array}{c}\text { Mass } \\
{\left[M_{\odot}\right]}\end{array}$ \\
\hline 18Sco & $5818 \pm 3$ & $4.457 \pm 0.010$ & $0.054 \pm 0.004$ & $1.01 \pm 0.01$ & $3.0 \pm 0.4$ & $1.040 \pm 0.002$ \\
HIP 56948 & $5793 \pm 6$ & $4.473 \pm 0.022$ & $0.019 \pm 0.005$ & $1.01 \pm 0.01$ & $2.6 \pm 1.0$ & $1.024 \pm 0.008$ \\
HIP 102152 & $5724 \pm 3$ & $4.357 \pm 0.009$ & $-0.013 \pm 0.003$ & $0.99 \pm 0.01$ & $8.0 \pm 0.3$ & $0.971 \pm 0.003$ \\
HD 20630 & $5747 \pm 8$ & $4.510 \pm 0.016$ & $0.065 \pm 0.007$ & $1.12 \pm 0.01$ & $1.4 \pm 0.6$ & $1.025 \pm 0.006$ \\
HD 202628 & $5836 \pm 7$ & $4.503 \pm 0.013$ & $0.009 \pm 0.005$ & $1.04 \pm 0.01$ & $0.8 \pm 0.6$ & $1.047 \pm 0.008$ \\
HIP 30502 & $5741 \pm 3$ & $4.435 \pm 0.008$ & $-0.053 \pm 0.003$ & $0.98 \pm 0.01$ & $5.7 \pm 0.4$ & $0.969 \pm 0.003$ \\
HIP 73815 & $5796 \pm 3$ & $4.320 \pm 0.008$ & $0.028 \pm 0.003$ & $1.07 \pm 0.01$ & $7.1 \pm 0.2$ & $1.012 \pm 0.004$ \\
HIP 77883 & $5702 \pm 3$ & $4.378 \pm 0.011$ & $0.023 \pm 0.003$ & $0.97 \pm 0.01$ & $7.5 \pm 0.4$ & $0.971 \pm 0.003$ \\
HIP 89650 & $5849 \pm 3$ & $4.403 \pm 0.011$ & $-0.013 \pm 0.003$ & $1.07 \pm 0.01$ & $5.1 \pm 0.5$ & $1.017 \pm 0.005$ \\
\hline
\end{tabular}

same approach described by Bedell et al. (2014), we measured in all the solar twin spectra and in the solar spectrum, the EWs of the absorption features listed in the master list. We detected in the spectra absorption features of 24 elements besides iron $(\mathrm{C}$, $\mathrm{O}, \mathrm{Na}, \mathrm{Mg}, \mathrm{Al}, \mathrm{Si}, \mathrm{S}, \mathrm{K}, \mathrm{Ca}, \mathrm{Sc}, \mathrm{Ti}, \mathrm{V}, \mathrm{Cr}, \mathrm{Mn}, \mathrm{Co}, \mathrm{Ni}, \mathrm{Cu}, \mathrm{Zn}$, $\mathrm{Y}, \mathrm{Ba}, \mathrm{La}, \mathrm{Ce}, \mathrm{Nd}, \mathrm{Eu})$.

We fed the qoyllur-quipu ${ }^{1}$ code with our EW measurements and it automatically estimated the stellar parameters by performing a line-by-line differential excitation/ionization balance analysis of the iron EWs relative to the solar spectrum. Namely, the $q^{2}$ code iteratively searched for the three equilibria (excitation, ionization, and the trend between the iron abundances and $\log (E W / \lambda))$. The iterations were executed with a series of steps starting from a set of initial parameters (i.e., the nominal solar parameters) and arriving at the final set of parameters that simultaneously fulfill the equilibria. Further details on this procedure can be found in Ramírez et al. (2014b). For our analysis we adopted the Kurucz (ATLAS9) grid of model atmospheres (Castelli \& Kurucz 2004) and we assumed the following solar parameters: $T_{\text {eff }}=5777 \mathrm{~K}, \log g=4.44 \mathrm{dex},[\mathrm{Fe} / \mathrm{H}]=0.00 \mathrm{dex}$, and $\xi=1.00 \mathrm{~km} \mathrm{~s}^{-1}$. The errors associated with the stellar parameters are evaluated by the code following the procedure described in Epstein et al. (2010) and Bensby et al. (2014). Since each atmospheric parameter is dependent on the others, this approach takes into account this dependence by propagating the error associated with the fulfillment of the three equilibrium conditions in every single parameter. The resulting stellar parameters and their uncertainties are reported in the first columns in Table 1.

Once the stellar parameters and the relative uncertainties have been determined for each star, $q^{2}$ automatically employs the appropriate atmospheric model for the local thermodynamic equilibrium (LTE) calculation of the chemical abundances through the 2014 version of MOOG (Sneden 1973). All the elemental abundances are normalized relative to the solar values on a line-by-line basis. The $q^{2}$ code calculates the error budget associated with the abundances $[\mathrm{X} / \mathrm{H}]$ taking into account the observational error due to the line-to-line scatter from the EW measurements (standard error) and the errors in the atmospheric parameters. When, as for $\mathrm{K}, \mathrm{Ce}, \mathrm{La}$, and $\mathrm{Eu}$, only one line is detected, the observational error is estimated by repeating the EW measurement five times with different assumptions on the continuum setting, adopting as error the standard deviation.

The $q^{2}$ code also calculates the non-LTE (NLTE) corrections for the oxygen abundances as a function of the stellar parameters

\footnotetext{
1 Qoyllur-quipu or $q^{2}$ (Ramírez et al. 2014b) is a Python package that is free and available online at https://gi thub.com/astroChasqui/ q2
}

assuming the NLTE corrections computed by Ramírez et al. (2007). As shown by Amarsi et al. (2016), for Sun-like stars these corrections are in good agreement with the more recent grids of NLTE corrections provided by Fabbian et al. (2009) and Amarsi et al. (2016). Nevertheless, we performed a quantitative test in order to evaluate how much our final oxygen abundances are affected by the choice of different grids of NLTE corrections. For this test we considered the two stars for which we expect the most extreme NLTE corrections to be applied: HIP 73815, which has the most discrepant $\log g$ from the solar value, and HIP 77883, which has the most discrepant $T_{\text {eff }}$ from the solar value. We found that the differential corrections in oxygen abundance between the grid of Amarsi et al. and that of Ramírez et al. is 0.003 dex for HIP 73815 and 0.002 dex for HIP $77883^{2}$. These quantities are much smaller than the uncertainties that we obtained for the oxygen abundances (i.e., $\geq 0.007 \mathrm{dex}$ ). For practical reasons we keep the oxygen abundances corrected for the Ramirez grid, since that grid is already implemented in the $q^{2}$ code.

In addition to this test, we verified on the same two stars whether NLTE corrections for additional elements should be taken into account. Based on the grid provided by Bergemann et al. (2012) we applied the differential NLTE corrections on 28 iron lines, which embody $\sim 30 \%$ of all the iron lines measured in the spectra of these stars. All other iron lines with EW measurements are not included in that grid. We found that differential NLTE effects are negligible for iron since it would account for a $[\mathrm{Fe} / \mathrm{H}]$ increment of $0.002 \mathrm{dex}$ in HIP 73815, while the iron abundance of HIP 77883 remained unaltered. We repeated the experiment for $\mathrm{Na}$ using the grid provided by Lind et al. (2011). For the star HIP 73815, the NLTE $\mathrm{Na}$ abundance differs from that obtained through MOOG by 0.003 dex. As for iron, no differences were seen for HIP 77883. Then we employed the grid described in Meléndez et al. (2016) to derive these differential corrections also for $\mathrm{Mg}$ and $\mathrm{Ca}$. Also for these two elements, the NLTE effects are negligible, since they are less than 0.005 and 0.004 dex for $\mathrm{Mg}$ and $\mathrm{Ca}$, respectively. These experiments ${ }^{3}$ show that the differential NLTE corrections for $\mathrm{Fe}, \mathrm{Na}, \mathrm{Mg}$, and $\mathrm{Ca}$ are small relative to uncertainties that we obtained for the abundances of these elements. As

2 The grid of Ramírez et al. establishes oxygen NLTE corrections of -0.022 dex for HIP 73815 and 0.000 dex for HIP 77883 , while the grid of Amarsi et al. predicts corrections of -0.025 and +0.002 dex, respectively.

3 The NLTE corrections for $\mathrm{Fa}$ and $\mathrm{Na}$ have been calculated through the interactive database INSPECT v1.0 available online at www. inspect-stars . com. For $\mathrm{Mg}$ and $\mathrm{Ca}$ we adopted an interpolator written for IDL. 
has been shown by other authors (Meléndez et al. 2012, 2016; Nissen 2015), differential NLTE effects in solar twins are negligible for all the other elements that we detected. According to this, oxygen is the only element that has been corrected for differential NLTE effects.

In addition, through the blends driver in the MOOG code, we took into account the hyperfine/isotopic splitting affecting the elements $\mathrm{Sc}, \mathrm{V}, \mathrm{Mn}, \mathrm{Co}, \mathrm{Cu}, \mathrm{Y}, \mathrm{Ba}, \mathrm{La}$, and $\mathrm{Eu}$. We assumed the HSF line list from the Kurucz database ${ }^{4}$.

Given the atmospheric parameters and their uncertainties, the $q^{2}$ code also calculates the age and mass probability distributions for each star according to the Yonsei-Yale isochrone set (Yi et al. 2001; Kim et al. 2002). Details on this procedure are given by Ramírez et al. (2013, 2014a).

The analysis described in this section allow us to determine the atmospheric parameters, ages, and masses for the nine solar twins observed through the UVES spectrograph. These values are listed in Table 1. The sample fully covers the age range of thin disk stars from 0.8 to $8.0 \mathrm{Gyr}$. The $[\mathrm{Fe} / \mathrm{H}]$ values are within -0.053 and 0.065 dex. The typical precisions that we reached are $\sigma\left(T_{\text {eff }}\right)=4 \mathrm{~K}, \sigma(\log g)=0.012 \operatorname{dex}, \sigma([\mathrm{Fe} / \mathrm{H}])=0.004$ dex, $\sigma(\xi)=0.01 \mathrm{~km} \mathrm{~s}^{-1}, \sigma($ Age $)=0.5 \mathrm{Gyr}$, and $\sigma($ Mass $)=0.005 M_{\odot}$. All the chemical abundances are listed in Table A.1.

Recently, Ramírez et al. (2012) analyzed the kinematics of seven solar twins of our sample establishing that they belong to the thin disk population. Assuming the parallaxes and proper motions from van Leeuwen (2007), and the radial velocities from Soubiran et al. (2013), we determined that HD 20630 and HIP 73815, the two stars in our sample not analyzed by Ramírez et al. (2012), have a probability $\geq 0.97$ of belonging to the thin disk population according to the membership criteria by Reddy et al. (2006).

\subsection{Data set from the literature}

We integrated the data set achieved from our UVES observations with the $[\mathrm{X} / \mathrm{Fe}]$ and age determinations provided by $\mathrm{N} 15$ and S16. The former ensured a data set of 18 thin disk stars and 3 high- $\alpha$-metal-rich stars, which are a population first identified by Adibekyan et al. (2011). As suggested by Haywood et al. (2013), this population is part of the thick disk and is characterized by higher $[\langle\alpha\rangle / \mathrm{Fe}]$ values $(\sim 0.1 \mathrm{dex})$ with respect to the normal thin disk stars. For simplicity, hereafter we will refer to the high- $\alpha$-metal-rich stars as "thick disk stars". For the star 18 Sco, which has been analyzed in the present work and by $\mathrm{N} 15$, we adopt the values determined here. We note that the agreement between our $[\mathrm{X} / \mathrm{Fe}]$ ratios and those of N15 is within 1 sigma with very high confidence given that the mean difference $\Delta[\mathrm{X} / \mathrm{Fe}](\mathrm{N} 15$; this work $)=0.006 \operatorname{dex}(\sigma=0.006 \mathrm{dex})$ and that only $[\mathrm{O} / \mathrm{Fe}]$ (out of 12 elements) have a difference that is marginally over the 1-sigma error.

In addition we included the data set from S16, who analyzed 13 thin disk and one thick disk star, determining ages and $[\mathrm{X} / \mathrm{Fe}]$ ratios. One star, HD 45184 , has been analyzed by both S16 and N15. As for 18 Sco, a high level of consistency resulted from the atmospheric parameters and abundance values determined for this star by N15 and S16, indicating that the data set provided by our group agrees with Nissen's data set. For HD 45184 we assumed, when available, the N15 values that are based on higher quality spectra, otherwise we used the $\mathrm{S} 16$ determinations.

\footnotetext{
4 The Kurucz database of atomic transitions is free and available at http://kurucz.harvard. edu/linelists.html
}

We also included in our data set the age and abundances determinations provided by Yana Galarza et al. (2016) for HIP 100963 and the abundances determined by Tucci Maia et al. (2014) for 16Cyg A and 16Cyg B. For 16Cyg A/B, we corrected the oxygen abundances for the NLTE effects through the same procedure described in Sect. 2. Since Tucci Maia et al. (2014) did not analyze the $n$-capture elements, the $[\mathrm{X} / \mathrm{Fe}]$ ratios of these elements, as well as the age determination of this system, have been taken from Ramírez et al. (2011).

Our final data set includes $[\mathrm{X} / \mathrm{Fe}]$ ratios and ages for 41 thin disk and four thick disk stars.

\section{3. $[\mathrm{X} / \mathrm{Fe}]$ vs. age relations}

As we mentioned in the introduction, chemical properties of stars provide extremely important insights into the mechanisms responsible for the formation and evolution of the Galaxy. In this section we first discuss the $[\mathrm{X} / \mathrm{Fe}]$ vs. age relations for the elements from $\mathrm{C}$ to $\mathrm{Zn}$ and then those for the $n$-capture elements (i.e., from $\mathrm{Y}$ to $\mathrm{Eu}$ ).

\subsection{Elements from $\mathrm{C}$ to $\mathrm{Zn}$}

The $[\mathrm{X} / \mathrm{Fe}]$ vs. age relations for the elements with $Z \leq 30$ are plotted in Fig. 1. We note that the $[\mathrm{X} / \mathrm{Fe}]$ ratios of most of the species are highly correlated with the stellar ages and that each element is characterized by its own relation. Figure 1 also shows that the thick disk stars do not necessarily follow the main distributions traced by the thin disk population. However, for some elements, like $\mathrm{Ca}$ and $\mathrm{Cr}$, they appear to follow the thin disk distribution. The difference between thin and thick disk stars is clearly visible in the $\alpha$-elements of which this thick disk population is systematically overabundant (as has been noted by Adibekyan et al. 2011; Haywood et al. 2013), but also in some iron-peak elements (such as $\mathrm{V}, \mathrm{Co}$, and $\mathrm{Ni}$ ), and in $\mathrm{Na}, \mathrm{Cu}$, and $\mathrm{Zn}$. For this reason we treat the thick disk stars separately from the sample of thin disk stars. As we can see, the boundary between the thin and thick disk populations is located at $\sim 8 \mathrm{Gyr}$ ago. This age is in perfect agreement with the formation age of the thin disk based on the G-dwarf metallicity distribution for stars in the solar vicinity (e.g., Chiappini et al. 1997).

From 8 Gyr towards younger ages, the chemical content of the thin disk evolved greatly through time, with each species undergoing different characteristic types of evolution. Each peculiar behavior must reflect the variety of the yields and timescales due to different sites of synthesis. Most of the [X/Fe]-age distributions seem non-linear and, in some cases, also non-monotonic. Only potassium does not exhibit a definite dependency on age, probably because its abundance determinations are only based on the saturated line at $7698.97 \AA$; and some weak telluric contamination may also be present.

\subsubsection{Fitting of the $[\mathrm{X} / \mathrm{Fe}]-$ age relations}

For all the elements, except potassium, we proceeded to fit the $[\mathrm{X} / \mathrm{Fe}]$ vs. age relations traced by the 41 thin disk stars assuming three different models and using the orthogonal distance regression method (Boggs \& Rogers 1990), which takes into account the uncertainties on both $[\mathrm{X} / \mathrm{Fe}]$ and ages ${ }^{5}$. First, we performed

5 For the data fitting we used the python scipy.odr package (http: //docs.scipy.org/doc/scipy/reference/odr.html). 
L. Spina et al.: Nucleosynthetic history of elements in the Galactic disk

\begin{tabular}{|lllll|}
\hline$-=$ Linear & $-\infty$ Two-segmented & $\$$ & Literature \\
\hline Hyperbolic & $\$-$ & This work & $\$$ & Thick disk \\
\hline
\end{tabular}
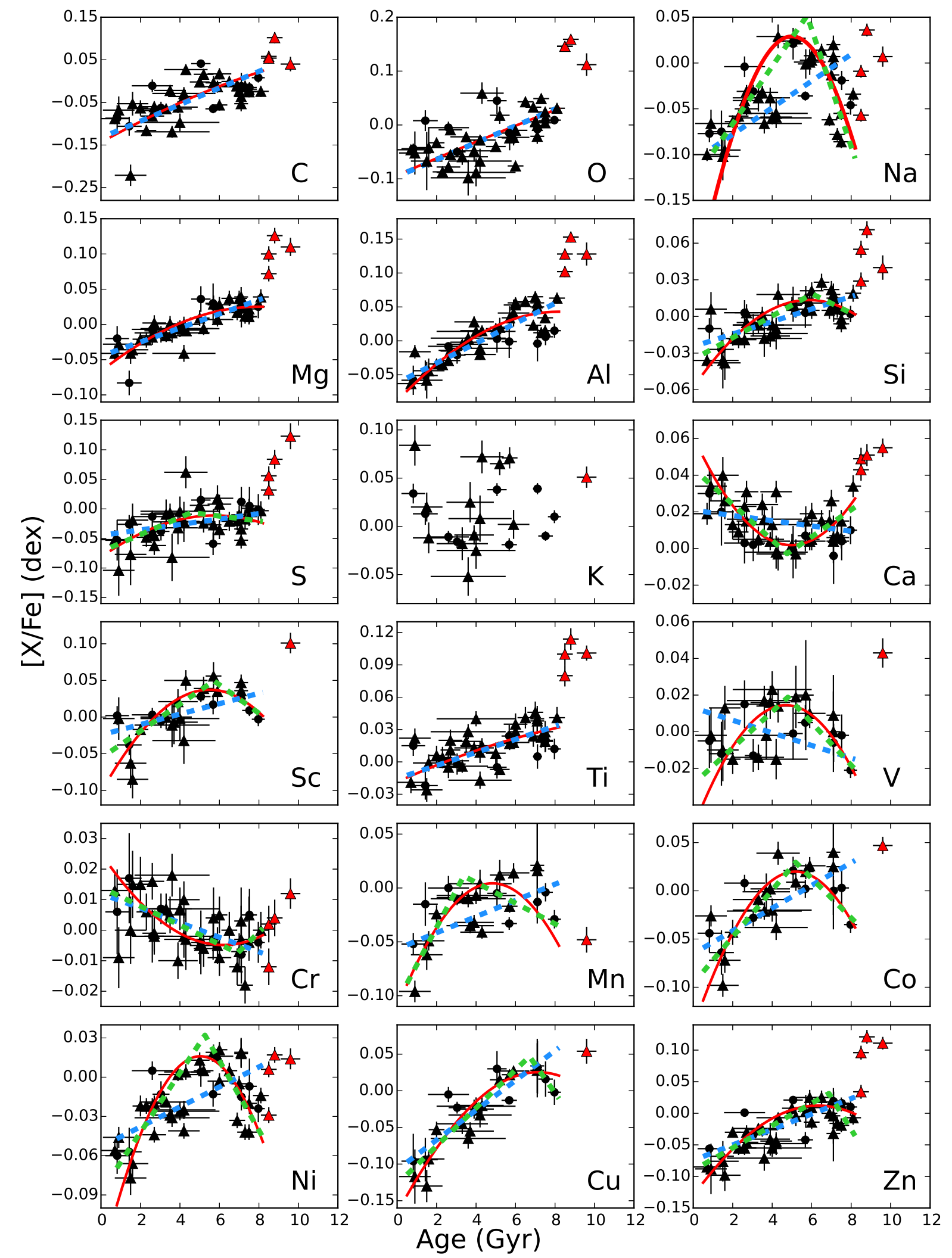

Fig. 1. $[\mathrm{X} / \mathrm{Fe}]$ ratios of the elements with $Z \leq 30$ as a function of stellar ages for the nine solar twins analyzed here (circles). We also plotted the measurements taken from the literature as triangles. The red triangles represent the thick disk stars. The $[\mathrm{X} / \mathrm{Fe}]$-age distributions have been fitted by linear, hyperbolic and two-segmented line functions showed as blue dashed, red solid and green dashed lines, respectively. The parameters related to these functions are listed in Table 2 . Note that the $y$-axes ranges are different from element to element, in order to properly visualize our entire data set. 
Table 2. Results of the fitting of the elements with $Z \leq 30$.

\begin{tabular}{|c|c|c|c|c|c|c|c|c|c|}
\hline \multirow[b]{2}{*}{ Element } & \multicolumn{2}{|c|}{ Linear } & \multicolumn{3}{|c|}{ Hyperbolic } & \multicolumn{4}{|c|}{ Two-segmented line } \\
\hline & $\begin{array}{c}a \\
{[\mathrm{dex}]}\end{array}$ & $\begin{array}{c}b \\
{\left[\operatorname{dex} \mathrm{Gyr}^{-1}\right]}\end{array}$ & $\begin{array}{c}k \\
{[\mathrm{Gyr}]}\end{array}$ & $\begin{array}{c}a \\
{[\mathrm{dex}]}\end{array}$ & $\begin{array}{c}b \\
{\left[\mathrm{Gyr} \mathrm{dex}^{-1}\right]}\end{array}$ & $\begin{array}{c}k \\
{[\mathrm{Gyr}]}\end{array}$ & $\begin{array}{c}a \\
{[\mathrm{dex}]}\end{array}$ & $\begin{array}{c}b \\
{\left[\operatorname{dex} \mathrm{Gyr}^{-1}\right]}\end{array}$ & $\begin{array}{c}c \\
{\left[\operatorname{dex} \mathrm{Gyr}^{-1}\right]}\end{array}$ \\
\hline $\mathrm{C}$ & $-0.133 \pm 0.018$ & $0.019 \pm 0.003$ & $27^{*}$ & $\sim 1.085$ & $\sim 22.8$ & - & - & - & - \\
\hline $\mathrm{O}$ & $-0.095 \pm 0.013$ & $0.016 \pm 0.002$ & $\gtrsim 7^{*}$ & $\sim 1.356$ & $\sim 45.8$ & - & - & - & - \\
\hline $\mathrm{Na}$ & $-0.107 \pm 0.019$ & $0.015 \pm 0.003$ & $5.0 \pm 0.1$ & $1.029 \pm 0.005$ & $6.3 \pm 0.4$ & $5.8 \pm 0.3$ & $-0.129 \pm 0.021$ & $0.0314 \pm 0.0076$ & $-0.064 \pm 0.017$ \\
\hline $\mathrm{Mg}$ & $-0.045 \pm 0.005$ & $0.0100 \pm 0.0009$ & $\gtrsim 7^{*}$ & $\sim 1.025$ & $\sim 18.2$ & - & - & - & - \\
\hline $\mathrm{Al}$ & $-0.062 \pm 0.008$ & $0.0147 \pm 0.0015$ & $\gtrsim 7^{*}$ & $\sim 1.043$ & $\sim 15.5$ & - & - & - & - \\
\hline $\mathrm{Si}$ & $-0.025 \pm 0.004$ & $0.0051 \pm 0.0008$ & $5.9 \pm 0.3$ & $1.013 \pm 0.002$ & $15.2 \pm 1.8$ & $6.0 \pm 0.4$ & $-0.035 \pm 0.005$ & $0.0089 \pm 0.0014$ & $-0.008 \pm 0.004$ \\
\hline S & $-0.045 \pm 0.009$ & $0.0046 \pm 0.0017$ & $5.8 \pm 0.6$ & $0.989 \pm 0.005$ & $15.1 \pm 3.6$ & $4.6 \pm 0.9$ & $-0.072 \pm 0.017$ & $0.0145 \pm 0.0062$ & $-0.004 \pm 0.005$ \\
\hline $\mathrm{Ca}$ & $0.021 \pm 0.004$ & $-0.0014 \pm 0.0008$ & $5.0 \pm 0.2$ & $-0.998 \pm 0.003$ & $14.2 \pm 1.4$ & $4.8 \pm 0.5$ & $0.044 \pm 0.007$ & $-0.0098 \pm 0.0025$ & $0.007 \pm 0.003$ \\
\hline $\mathrm{Sc}$ & $-0.024 \pm 0.010$ & $0.0071 \pm 0.0018$ & $5.5 \pm 0.2$ & $1.037 \pm 0.005$ & $10.0 \pm 1.0$ & $5.7 \pm 0.5$ & $-0.056 \pm 0.013$ & $0.0184 \pm 0.0037$ & $-0.019 \pm 0.009$ \\
\hline $\mathrm{Ti}$ & $-0.016 \pm 0.005$ & $0.0061 \pm 0.0011$ & $\gtrsim 7^{*}$ & $\sim 1.045$ & $\sim 41.2$ & - & - & - & - \\
\hline $\mathrm{V}$ & $0.013 \pm 0.007$ & $-0.0034 \pm 0.0012$ & $4.7 \pm 0.2$ & $1.014 \pm 0.003$ & $12.6 \pm 1.2$ & $4.8 \pm 0.5$ & $-0.029 \pm 0.009$ & $0.0099 \pm 0.0031$ & $-0.012 \pm 0.003$ \\
\hline $\mathrm{Cr}$ & $0.012 \pm 0.003$ & $-0.0024 \pm 0.0005$ & $6.2 \pm 0.7$ & $-1.005 \pm 0.002$ & $25.0 \pm 5.1$ & $6.9 \pm 0.5$ & $0.014 \pm 0.003$ & $-0.0031 \pm 0.0007$ & $0.006 \pm 0.006$ \\
\hline $\mathrm{Mn}$ & $-0.057 \pm 0.010$ & $0.0075 \pm 0.0019$ & $4.8 \pm 0.2$ & $1.004 \pm 0.008$ & $9.8 \pm 1.5$ & $3.4 \pm 0.7$ & $-0.106 \pm 0.041$ & $0.0347 \pm 0.0187$ & $-0.010 \pm 0.005$ \\
\hline Co & $-0.065 \pm 0.015$ & $0.012 \pm 0.003$ & $5.2 \pm 0.2$ & $1.020 \pm 0.004$ & $8.8 \pm 0.7$ & $5.2 \pm 0.3$ & $-0.096 \pm 0.014$ & $0.0242 \pm 0.0040$ & $-0.020 \pm 0.006$ \\
\hline $\mathrm{Ni}$ & $-0.053 \pm 0.010$ & $0.0076 \pm 0.0017$ & $5.0 \pm 0.1$ & $1.016 \pm 0.004$ & $8.6 \pm 0.6$ & $5.3 \pm 0.4$ & $-0.088 \pm 0.015$ & $0.0228 \pm 0.0052$ & $-0.027 \pm 0.006$ \\
\hline $\mathrm{Cu}$ & $-0.108 \pm 0.013$ & $0.020 \pm 0.003$ & $7.1 \pm 0.8$ & $1.025 \pm 0.008$ & $11.0 \pm 1.8$ & $6.7 \pm 0.5$ & $-0.127 \pm 0.014$ & $0.0260 \pm 0.0033$ & $-0.039 \pm 0.037$ \\
\hline $\mathrm{Zn}$ & $-0.075 \pm 0.007$ & $0.0122 \pm 0.0014$ & $6.3 \pm 0.4$ & $1.012 \pm 0.004$ & $11.4 \pm 1.4$ & $6.9 \pm 0.3$ & $-0.090 \pm 0.009$ & $0.0177 \pm 0.0021$ & $-0.051 \pm 0.035$ \\
\hline
\end{tabular}

Notes. ${ }^{(*)}$ The values used for the functions plotted in Fig. 1 are 16.3, 48.0, 8.0, 8.2, and 15.0 Gyr for C, O Mg, Al, and Ti, respectively.

the fit employing a straight line function, defined as

$\left[\frac{\mathrm{X}}{\mathrm{Fe}}\right]=a+b \times \mathrm{Age}[\mathrm{Gyr}]$

This very simple model has only two parameters, and it has also been assumed by N15 and S16 for their data fitting. The final parameters and their uncertainties are listed in the second and third columns of Table 2 and plotted in Fig. 1 as blue dashed lines. We note that these slopes are in agreement with those of N15 within their uncertainties for all the elements, except for carbon. In the first three columns of Table 3, we list for each element the average of the residuals $\left(\sigma_{[\mathrm{X} / \mathrm{Fe}]}\right)$ and the reduced chi-square $\left(\chi_{\mathrm{red}}^{2}\right)$ obtained from the linear fitting. From these values we note that some elements, such as $\mathrm{Mg}$ and $\mathrm{Cr}$, have $\sigma_{[\mathrm{X} / \mathrm{Fe}]}$ values that are smaller than typical uncertainties on their $[\mathrm{X} / \mathrm{Fe}]$ ratios and that have $\chi_{\text {red }}^{2} \sim 1$. On the other hand, the straight line model completely fails to fit the $[\mathrm{X} / \mathrm{Fe}]-$ age relations of $\mathrm{Na}$ and Ni. Similar differences in the fit goodness have also been found by N15 when comparing the same elements. As mentioned in the introduction, $\mathrm{S} 16$ attributed this large dissimilarity to turnovers that may be present in the $[\mathrm{X} / \mathrm{Fe}]-$ age distributions.

In order to verify whether these relations are linear or whether they have turnovers as suggested by S16, we repeated the fitting procedure using a model based on a general hyperbolic function:

$\left[\frac{\mathrm{X}}{\mathrm{Fe}}\right]=a \pm \sqrt{\frac{(\mathrm{Age}[\mathrm{Gyr}]-k)^{2}}{b^{2}}+1}$

This model has three parameters and it would provide rough, but adequate estimations of the ages correspondent to the turnovers or stationary points (hereafter "knees") of the $[\mathrm{X} / \mathrm{Fe}]$ vs. age distributions (the " $k$ " parameter in the equation above). The resulting parameters are reported in the fourth, fifth, and sixth columns of Table 2 and plotted in Fig. 1 as solid red lines. The parameters of the hyperbolic functions are satisfactorily constrained; the only exceptions are $\mathrm{C}, \mathrm{O}, \mathrm{Mg}, \mathrm{Al}$, and $\mathrm{Ti}$, whose $[\mathrm{X} / \mathrm{Fe}]$ ratios show a quasi-linear dependence with age. For these elements we list in Table 2 a lower limit for the $k$ parameter at 7 Gyr. Comparing the $\chi_{\text {red }}^{2}$ of the linear fit to those of hyperbolic fit reported in Table 3, we note that the hyperbolic model resulted in lower $\chi_{\text {red }}^{2}$ values for the majority of the elements, except for $\mathrm{C}, \mathrm{O}, \mathrm{Al}$, and $\mathrm{Ti}$.

The third model used to fit the data set is based on a twosegmented function, defined as

$$
\left[\frac{\mathrm{X}}{\mathrm{Fe}}\right]= \begin{cases}a+b \times \mathrm{Age}[\mathrm{Gyr}] & \text { if Age }[\mathrm{Gyr}] \leq k \\ a+(b-c) \times k+c \times \operatorname{Age}[\mathrm{Gyr}] & \text { if } \mathrm{Age}[\mathrm{Gyr}]>k\end{cases}
$$

We did not perform this fit on the elements whose parameters have not been strongly constrained through the hyperbolic model (i.e., $\mathrm{C}, \mathrm{O}, \mathrm{Mg}, \mathrm{Al}$, and $\mathrm{Ti}$ ), since the fitting procedure would result in functions very similar to straight lines. The parameters are listed in the last four columns of Table 2 and the functions are plotted in Fig. 1 as dashed green lines. The two-segmented line model is the most complex of the three models adopted for the fitting procedure (it has four parameters), but it allows us to verify whether we can recover knee-ages similar to those obtained through the hyperbolic model. The values of the k parameter obtained from the two models are consistent within the uncertainties, except for $\mathrm{Na}$ and $\mathrm{Mn}$.

In order to evaluate which of the three models provides the best fit of the $[\mathrm{X} / \mathrm{Fe}]-$ age relations, we conducted a statistical comparison of the results based on the $F$ ratio (Fisher 1925). 
Table 3. Statistical tests resulting from the fitting of the elements with $Z \leq 30$.

\begin{tabular}{c|ccc|cc|cc|cc|cc}
\hline \hline & \multicolumn{3}{|c|}{ Linear } & \multicolumn{2}{|c|}{ Hyperbolic } & \multicolumn{2}{|c|}{ Two-segmented line } & \multicolumn{3}{c}{ Statistical model comparison } \\
\hline Element & $\begin{array}{c}\sigma_{[\mathrm{X} / \mathrm{Fe}]} \\
{[\mathrm{dex}]}\end{array}$ & $\chi_{\text {red }}^{2}$ & $\mathrm{df}^{*}$ & $\begin{array}{c}\sigma_{[\mathrm{X} / \mathrm{Fe}]} \\
{[\mathrm{dex}]}\end{array}$ & $\chi_{\text {red }}^{2}$ & $\begin{array}{c}\sigma_{[\mathrm{X} / \mathrm{Fe}]} \\
{[\mathrm{dex}]}\end{array}$ & $\chi_{\text {red }}^{2}$ & $F_{\text {Lin-Hyp }}$ & $P_{\text {Lin-Hyp }}$ & $F_{\text {Lin-2SL }}$ & $P_{\text {Lin-2SL }}$ \\
\hline $\mathrm{C}$ & 0.033 & 4.86 & 39 & 0.033 & 5.04 & - & - & -0.37 & - & - & - \\
$\mathrm{O}$ & 0.028 & 5.19 & 39 & 0.028 & 5.37 & - & - & -0.27 & - & - & - \\
$\mathrm{Na}$ & 0.030 & 12.46 & 39 & 0.033 & 1.89 & 0.028 & 1.98 & 218.94 & $1.11^{-16}$ & 106.20 & $4.44^{-16}$ \\
$\mathrm{Mg}$ & 0.011 & 1.04 & 39 & 0.011 & 0.91 & - & - & 6.22 & $1.71^{-2}$ & - & - \\
$\mathrm{Al}$ & 0.017 & 2.56 & 39 & 0.015 & 2.61 & - & - & 0.21 & - & - & - \\
$\mathrm{Si}$ & 0.010 & 1.60 & 39 & 0.010 & 1.16 & 0.009 & 1.14 & 15.8 & $3.03^{-4}$ & 8.54 & $8.95^{-4}$ \\
$\mathrm{~S}$ & 0.020 & 1.51 & 38 & 0.021 & 1.40 & 0.020 & 1.33 & 3.97 & $5.36^{-2}$ & 3.33 & $4.70^{-2}$ \\
$\mathrm{Ca}$ & 0.009 & 2.23 & 39 & 0.009 & 1.28 & 0.008 & 1.34 & 30.01 & $2.89^{-6}$ & 14.19 & $2.66^{-5}$ \\
$\mathrm{Sc}$ & 0.021 & 2.67 & 23 & 0.021 & 1.20 & 0.019 & 1.33 & 29.19 & $2.00^{-5}$ & 13.37 & $1.80^{-4}$ \\
$\mathrm{Ti}$ & 0.012 & 2.13 & 39 & 0.012 & 2.21 & - & - & -0.40 & - & - & - \\
$\mathrm{V}$ & 0.014 & 1.92 & 23 & 0.011 & 0.85 & 0.011 & 0.82 & 29.84 & $1.73^{-5}$ & 15.12 & $8.56^{-5}$ \\
$\mathrm{Cr}$ & 0.006 & 1.03 & 39 & 0.006 & 0.96 & 0.006 & 0.93 & 3.57 & $6.64^{-2}$ & 2.87 & $6.94^{-2}$ \\
$\mathrm{Mn}$ & 0.019 & 4.14 & 23 & 0.019 & 2.26 & 0.020 & 2.71 & 20.17 & $1.82^{-4}$ & 8.08 & $2.50^{-3}$ \\
$\mathrm{Co}$ & 0.021 & 4.39 & 23 & 0.020 & 0.96 & 0.020 & 1.06 & 83.68 & $5.95^{-9}$ & 39.36 & $7.87^{-8}$ \\
$\mathrm{Ni}$ & 0.018 & 7.51 & 39 & 0.019 & 1.61 & 0.017 & 1.77 & 143.85 & $1.73^{-14}$ & 68.77 & $3.44^{-13}$ \\
$\mathrm{Cu}$ & 0.019 & 1.62 & 23 & 0.017 & 1.21 & 0.013 & 1.05 & 8.94 & $6.74^{-3}$ & 6.03 & $8.53^{-3}$ \\
$\mathrm{Zn}$ & 0.019 & 2.34 & 39 & 0.018 & 1.80 & 0.019 & 1.55 & 12.70 & $1.01^{-3}$ & 9.17 & $5.88^{-4}$ \\
\hline
\end{tabular}

Notes. ${ }^{(*)}$ The degrees of freedom (df) are listed only for the linear fitting (two parameters). Those related to the hyperbolic (three parameters) and two-segmented line (four parameters) fitting are immediately deducible.

This quantity associates the relative decrease in $\chi^{2}$ due to the use of two different models in the fitting procedure with the relative decrease in degrees of freedom. Namely, the $F$ ratio is defined as

$$
F=\frac{\left(\chi_{1}^{2}-\chi_{2}^{2}\right) / \chi_{2}^{2}}{\left(d f_{1}-d f_{2}\right) / d f_{2}},
$$

where $\chi_{1}^{2}$ and $d f_{1}$ are the chi-square and the degree of freedom of the simplest model. Thus, when $F>1$ the relative decrease in $\chi^{2}$ is greater than the relative decrease in degrees of freedom. There are two possible explanations for this case: either the more complex model is the best or the simpler model is correct, but random scatter makes the more complex model fitting better. In this instance, the knowledge of $P$ values allows us to identify which is the correct explanation, since it represents the probability that a random scatter is dominating the fitting (Pearson 1900; Fisher 1925). In the last four columns of Table 3 we list the $F$ ratios and the $P$ values obtained by comparing the linear model with the hyperbolic model (columns eight and nine) and the linear model with the two-segmented line model (columns ten and eleven). We did not calculate the $P$ value when $F<1$. These quantities clearly indicate that the two more complex models (i.e., the hyperbolas and the two-segmented lines) reproduce the data set better, except for $\mathrm{C}, \mathrm{O}, \mathrm{Al}$, and $\mathrm{Ti}$, which have $\mathrm{F}$ ratios $<1$. Among the 13 elements with $F_{\text {Lin-Hyp }}>1$, all the species have $P_{\text {Lin-Hyp }}<0.07$ and only three have $P_{\text {Lin-Hyp }}>0.01$ (i.e., $\mathrm{Mg}, \mathrm{S}$, and $\mathrm{Cr}$ ). Similar results have been found when comparing the straight line model with the two-segmented line model. The outcomes of this statistical comparison between the three models demonstrates that indeed most of the $[\mathrm{X} / \mathrm{Fe}]-$ age relations analyzed in this paper are characterized by a knee located at different ages between 4 and 7 Gyr. As we detail in Sect. 3.1.2, the knee ages depend on the nature of the specific element. In addition, $F_{\text {Lin-Hyp }}$ is always greater than $F_{\text {Lin-2SL }}$, which indicates that the hyperbolic model is the function that fits the data better between the two non-linear models. For this reason, hereafter we discuss only the parametrization of the $[\mathrm{X} / \mathrm{Fe}]-$ age relations defined through the hyperbolic model.

\subsubsection{Evolution of the $[\mathrm{X} / \mathrm{Fe}]$ ratios}

The hyperbolic functions overplotted in Fig. 1 and parametrized in Table 2 highlight the peculiarities of each element in the $[\mathrm{X} / \mathrm{Fe}]$ vs. age relations that reflect their various histories of nucleosynthesis. We note that most of the elements are characterized by an early $[\mathrm{X} / \mathrm{Fe}]$ increment with time that is followed by a decrement for the youngest stars (e.g., $\mathrm{Na}$ and $\mathrm{Ni}$ ). The age of the knee can vary significantly from 4 Gyr to older ages. Some other species (i.e., $\mathrm{O}, \mathrm{Mg}, \mathrm{Al}$, and $\mathrm{Ti}$ ) do not show the early increment; however, their distributions flatten at 6-8 Gyr. Only $\mathrm{Ca}$ and $\mathrm{Cr}$ present an early $[\mathrm{X} / \mathrm{Fe}]$ decrement with time and a subsequent rise.

Assuming the yields from SNe II and SNe Ia (W7 model) listed in Nomoto et al. (1997a,b), we plot in Fig. 2 the knee-ages of the various elements as a function of the ratios between the yields of the two types of SNe. Interestingly, most of the elements that are mainly produced by $\mathrm{SNe}$ II (i.e., the $\alpha$-elements) are those with a knee-age $\gtrsim 6$ Gyr. Instead, all the elements yielded primarily by $\mathrm{SNe}$ Ia (i.e., the iron-peak elements) have knee-ages $<5.5$ Gyr.

As mentioned in the introduction, the $\alpha$-elements are produced primarily by $\mathrm{SNe}$ II events due to high-mass stars of negligible lifetimes in comparison to galaxy evolution timescales. On the other hand, iron is spread into the ISM by both SNe II and $\mathrm{SNe}$ Ia, where SNe Ia operate on longer timescales. Therefore, the oldest stars of the thin disk are those formed shortly after the ISM has been enriched by SNe II, thus they should show higher $[\alpha / \mathrm{Fe}]$ ratios. In addition, the stars that formed sometime after the SNe Ia pollution will have higher iron abundances and lower $[\alpha / \mathrm{Fe}]$ ratios. As can be seen in Fig. 1, the decrease in the $[\alpha / \mathrm{Fe}]$ ratios began at very old ages, at $\sim 7 \mathrm{Gyr}$ or even earlier. This would suggest that the enrichment from SNe Ia started to be 


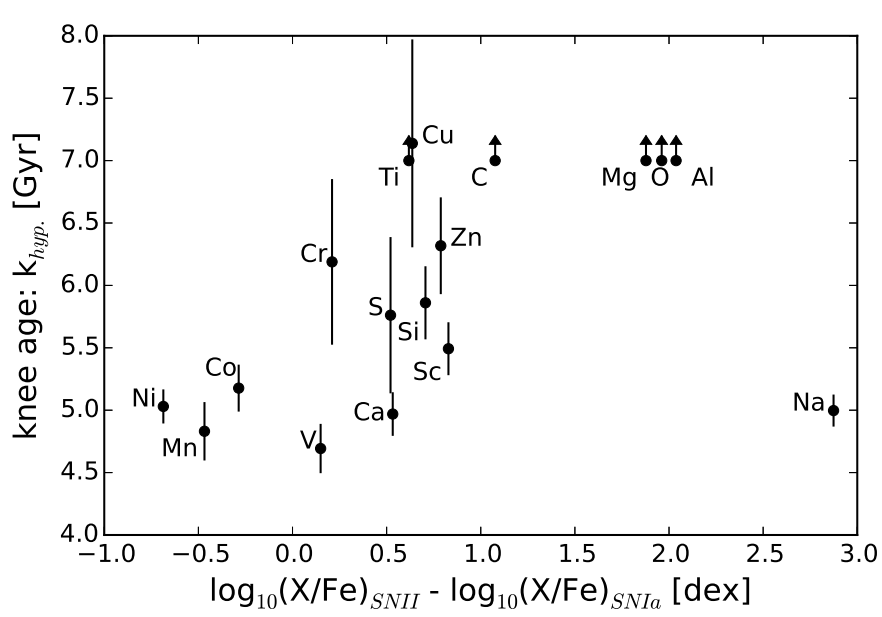

Fig. 2. Ages for the location of the knee in the $[\mathrm{X} / \mathrm{Fe}]-$ age relations (i.e., the $\mathrm{k}$ parameters from the hyperbolic fitting in Table 2) as a function of logarithmic ratios between the yields of SNe II and $\mathrm{SNe}$ Ia (Nomoto et al. 1997a,b).

significant not later than $\sim 1$ Gyr after the formation of the thin disk.

The unique element that does not fit the explanation is $\mathrm{Na}$, which - like the $\alpha$-elements - is mainly produced by SNe II (see Fig. 2), but its knee-age value is similar to that typical of ironpeak elements ( $\sim 5 \mathrm{Gyr})$. This peculiar behavior is somewhat expected, since the synthesis of $\mathrm{Na}$ is controlled by the neutron excess (Timmes et al. 1995), meaning that the $\mathrm{Na}$ mass produced in SNe is highly dependent on the stellar metallicity (see Fig. 5 in Kobayashi et al. 2006). Thus, the first generation of stars, those with the lowest metallicity, did not deliver as much $\mathrm{Na}$ to the ISM as the later SNe progenitor did. This would explain why the thin disk underwent a gradual $[\mathrm{Na} / \mathrm{Fe}]$ increase during its first 2-3 Gyr, with a knee-age that is somewhat delayed in time with respect to that of other $\alpha$-elements such as $\mathrm{Si}$ or S. Analogously to $\mathrm{Na}, \mathrm{Sc}$ is also mainly synthesized by $\mathrm{SNe}$ II, with a high dependency on the metallicity of the progenitor (Kobayashi et al. 2006): the mass of Sc produced by stars with solar metallicity $(Z=0.02)$ is more than ten times the mass produced by stars with $Z=0$. Also for this element we observe a knee that is slightly delayed in comparison with other $\alpha$-elements.

Since the enrichment from massive stars lasts only while the stellar formation is active, the rate of SNe II is expected to decrease after the first few Gyrs from the formation of the Galactic disk (Gibson et al. 2003), quenching their contribution to the pollution of the ISM. On the other hand, iron is continuously delivered by SNe Ia resulting in a decrement of the $[\alpha / \mathrm{Fe}]$ ratios through younger ages.

Like iron, the iron-peak elements $\mathrm{V}, \mathrm{Mn}, \mathrm{Co}$, and $\mathrm{Ni}$ are produced by both $\mathrm{SNe}$ II and $\mathrm{SNe}$ Ia, and their $[\mathrm{X} / \mathrm{Fe}]$ vs. age relations are similar to that displayed by $\mathrm{Na}$ : an early $[\mathrm{X} / \mathrm{Fe}]$ rise, the knee at $\sim 5 \mathrm{Gyr}$, and a later fall towards younger ages (see Fig. 1). For these elements, the $[\mathrm{X} / \mathrm{Fe}]$ increment extended in time likely reflects the longer timescales in which SNe Ia pollute the ISM. However, the reason for the knee and the late $[\mathrm{X} / \mathrm{Fe}]$ decrease are still unclear. The significant and in some cases dominant contribution from $\mathrm{SNe}$ Ia toward the production of these elements excludes a simple explanation, mainly because of the variety of the possible SN Ia sources.

A series of observational studies makes it clear that both the environment and the path that lead to SNe Ia explosions play a role in their yields (e.g., Stritzinger et al. 2006; Ellis et al. 2008; Howell et al. 2009; Kelly et al. 2010; Foley et al. 2013; Graham et al. 2015). These results corroborate the outcomes of several theoretical models suggesting that the $\mathrm{SNe}$ Ia yields are strongly affected by the metallicity, mass, and age of the progenitor (e.g., Timmes et al. 2003; Travaglio et al. 2005, 2015; Mannucci et al. 2006; Jackson et al. 2010; De et al. 2014) and by asymmetries in the explosion (Chamulak et al. 2012).

It is plausible that the late evolution of the iron-peak elements could be the result of the two different main channels that trigger the SN Ia explosions: the single- and the doubledegenerate scenarios (Iben \& Tutukov 1984; Webbink 1984). A key difference, for example, is the fact that the two channels involve very different time distributions of the SNe rates (e.g., Yungelson \& Livio 2000; Ruiter et al. 2009; Mennekens et al. 2010; Yungelson 2010; Bours et al. 2013; Wang et al. 2013; Claeys et al. 2014; Maoz et al. 2014). Namely, for the singledegenerate model, most of the possible donors ${ }^{6}$ are limited in a strict range of masses, $\sim 2-3 M_{\odot}$ (Langer et al. 2000; Han \& Podsiadlowski 2004), and since these systems explode shortly after the donor evolves out of the main sequence, the resulting SNe Ia explosions are mostly concentrated in a narrow interval of time between a few hundred Myrs and 2 Gyr after the onset of star formation. On the other hand, in addition to the prompt SNe Ia explosions proposed by Bonaparte et al. (2013), the merger rate in the double-degenerate models is expected to reach a maximum very early (the typical time in which the stars with masses below $8 M_{\odot}$ evolve into the first white dwarfs) and then moderately declines with time. This implies that the production of iron-elements in the early evolution of the Galaxy should be driven by both of the SN Ia channels, but after $\sim 5 \mathrm{Gyr}$ from the initial burst of star formation it should be dominated by $\mathrm{SNe}$ Ia caused by the merger of white dwarfs.

It is also very likely that the exploding white dwarf mass in the double-degenerate model might play an important role in the chemical evolution of the Galactic disk. In addition, it is generally accepted that, under certain circumstances, the SN Ia detonation can be activated even if the combined mass does not exceed the Chandrasekhar mass (e.g., Pakmor et al. 2010; van Kerkwijk et al. 2010). Owing to the lower mass of the progenitors, this $s u b$-Chandrasekhar mass scenario could be of great importance in the latest evolution of the Galaxy. It is also remarkable that, as shown by Seitenzahl et al. (2013), the material released into the ISM by the sub-Chandrasekhar SN Ia have a $[\mathrm{Mn} / \mathrm{Fe}]$ ratio that is negative and significantly lower than that produced by near-Chandrasekhar SN Ia explosions. This would imply a late decrement of the $[\mathrm{Mn} / \mathrm{Fe}]$ ratio with time, similar to what we observe in Fig. 1.

Finally, the recent calculations performed by Miles et al. (2016) have shown that the variations in the progenitor metallicity results in several trends in the SNe Ia yields since they are affected by the neutron excess in the composition of the progenitor white dwarf. Namely, the increase in the initial metallicity would lead to a more efficient production of iron (see Fig. 3 in Miles et al. 2016). This could account for the lower $[\mathrm{X} / \mathrm{Fe}]$ ratios observed for the iron-peak elements in the youngest stars.

\footnotetext{
6 The single-degenerate scenario for near-Chandrasekhar mass white dwarfs has at least two sub-channels dependent on the mass of the donor (Kobayashi et al. 1998). In the first class helium-burning intermediatemass stars donate mass to the white dwarf, while in the second class the donor is a red giant or main-sequence star of $\sim 1 M_{\odot}$. The first class has shorter timescales ( 100-500 Myr) and is the dominant channel, while the second has timescales $>1$ Gyr (Ruiter et al. 2014).
} 




Fig. 3. $[\mathrm{Ni} / \mathrm{Fe}]$ as a function of $[\mathrm{Na} / \mathrm{Fe}]($ left $)$ and as a function of $[\mathrm{Sc} / \mathrm{Fe}]($ right $)$, with the symbols as in Fig. 1. The dashed red lines correspond to linear fits.

Additional hints regarding the production of an iron-peak element like Ni could be provided by the plot in Fig. 3 (left) showing that there is a tight relation between the $[\mathrm{Na} / \mathrm{Fe}]$ and $[\mathrm{Ni} / \mathrm{Fe}]$ ratios. A linear fit that takes into account the errors in both coordinates provides the following relation

$\left[\frac{\mathrm{Ni}}{\mathrm{Fe}}\right]=0.0021( \pm 0.0018)+0.58( \pm 0.04) \times\left[\frac{\mathrm{Na}}{\mathrm{Fe}}\right]$

with a standard deviation of $\sigma_{[\mathrm{Ni} / \mathrm{Fe}]}=0.008$ dex. This correlation has already been seen among solar twin stars (N15) and for stars in dwarf spheroidal galaxies (Venn et al. 2004; Letarte et al. 2010; Lemasle et al. 2014). As enlightened by Venn et al. (2004), ${ }^{23} \mathrm{Na}$ is the only stable neutron-rich isotope produced in large amounts by the hydrostatic $\mathrm{C}$ burning during the SN II events. The stable ${ }^{58} \mathrm{Ni}$ isotope is also yielded during the SN II explosions, but its production is controlled by the abundance of the neutron-rich elements like ${ }^{23} \mathrm{Na}$ (for more details, see Thielemann et al. 1990). This is why a Na-Ni relation is expected when the chemical enrichment is dominated by SN II explosions. However, a significant amount of $\mathrm{Ni}$ is also produced by SN Ia explosions, whose Na yields are not well constrained. This is why it is still unclear whether there is a contribution of $\mathrm{SNe}$ Ia to the Na-Ni relation. Our data clearly show that such relations exist for stars of all ages and of the two populations (thin and thick disk), which may suggests that the channels that are leading the latest chemical evolution of the Galactic disk are maintaining the $\mathrm{Na}-\mathrm{Ni}$ dependence.

Other elements, such as Sc, show similar correlations with $\mathrm{Ni}$ (Fig. 3, right) owing to the neutron-rich environment that boosts the production of both the elements (e.g., Kobayashi et al. 2006). However, the Sc-Ni correlation is not as tight as that found for $\mathrm{Na}-\mathrm{Ni}$. In addition, contrary to the $\mathrm{Na}-\mathrm{Ni}$ relation, the thick disk star is an outlier of the Sc-Ni main distribution and, for this reason, has not been considered for the linear fit.

In addition, the mechanism that is driving the increase in the $[\mathrm{Ca} / \mathrm{Fe}]$ and $[\mathrm{Cr} / \mathrm{Fe}]$ ratios seen for the youngest stars is still an open question. As is true for the iron-peak elements, the uncertainties of the models and the wide variety of the SNe Ia explosion mechanisms very likely complicate a simple explanation. For instance, as suggested by N15, the increasing trend with time observed for Ca could be the effect of a new type of SNe characterized by a large production of $\mathrm{Ca}$ that would be up to 10 times greater than that synthesized by SN Ia (Perets et al. 2010).

\subsection{Neutron-capture elements}

The $[\mathrm{X} / \mathrm{Fe}]$ ratios of the $n$-capture elements are plotted in Fig. 4 as a function of the stellar ages. As mentioned in Sect. 1, these species are synthesized by a mixture of the $s$ - and $r$-process. The thin disk stars are characterized by a $[\mathrm{X} / \mathrm{Fe}]$ increment with time, which is probably the result of the elemental synthesis from the $s$-process in AGB stars. In fact, the latest contribution to chemical evolution by AGB stars, especially those of lower mass (Busso et al. 2001; D'Orazi et al. 2009; Maiorca et al. 2011), should have an impact on the production $n$-capture elements greater than that of SNe (Battistini \& Bensby 2016). Therefore, the $[\mathrm{X} / \mathrm{Fe}]$-age dependency of each $n$-element is probably mainly controlled in the thin disk by its rate of production through the $s$-process rather than the $r$-process.

With the aim of testing this hypothesis, we performed a linear fit of the $[\mathrm{X} / \mathrm{Fe}]$ vs. age relations shown by the thin disk stars in Fig. 4, adopting the orthogonal distance regression method and the following formula, $[\mathrm{X} / \mathrm{Fe}]=a+b \times$ age. The resulting parameters are listed in Table 4 and the linear fits are overplotted in Fig. 4. In Fig. 5 we show the slope of each element (i.e., the $b$ parameters) obtained by the linear fitting as a function of their solar $s$-process contribution percentages taken from Bisterzo et al. (2014). Based on these production rates, we can consider $\mathrm{Ba}$ as a prototype $s$-process element owing to its high $s$-process contribution, which is responsible for $\sim 81 \%$ of $\mathrm{Ba}$. On the other hand, Eu can be considered a nearly pure $r$-process element, since the $s$-process accounts for only $\sim 6 \%$ of its synthesis. The difference in the production rates through the $s$-process between these two elements results in two very different $b$ slopes: Ba has a very steep $[\mathrm{X} / \mathrm{Fe}]$-age dependence, while the correlation found for $\mathrm{Eu}$ is almost flat. Indeed, a relation between the $b$ parameters and the $s$-process contributions exists in Fig. 5 for all the $n$-capture elements; namely, the elements with a higher contribution from the $s$-process are those with a steeper $[\mathrm{X} / \mathrm{Fe}]$-age slope. This result is consistent with AGBs being mainly responsible for the 

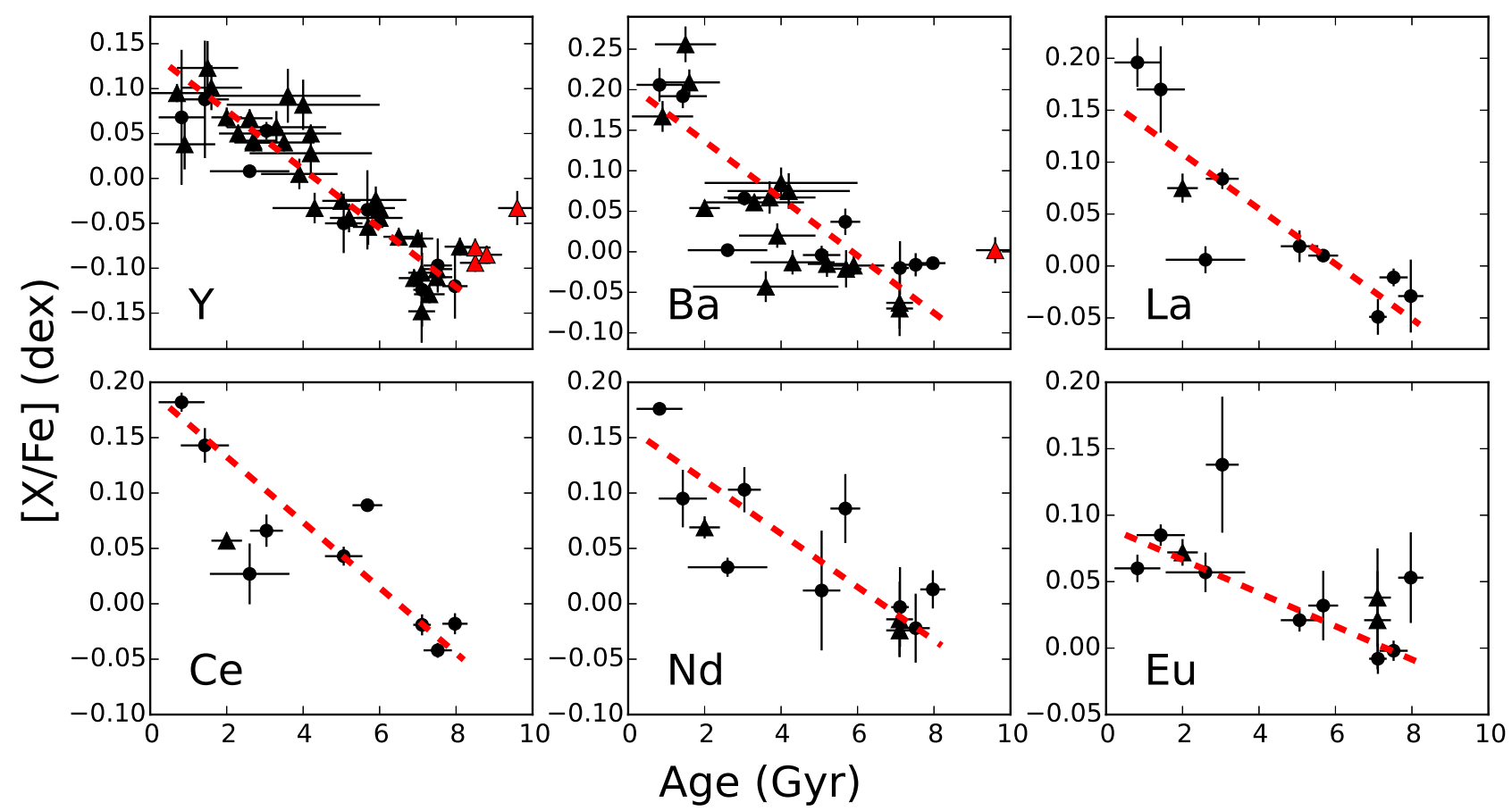

Fig. 4. $[\mathrm{X} / \mathrm{Fe}]$ ratios as a function of stellar ages for the nine solar twins analyzed here (circles). We also plotted the measurements from Ramírez et al. (2011), Nissen (2015), Spina et al. (2016), Yana Galarza et al. (2016), as triangles. The red triangles represent the thick disk stars. The $[\mathrm{X} / \mathrm{Fe}]$-age distributions have been fitted by the functions showed as red dashed lines, whose parameters are listed in Table 4.

Table 4. Results of the fitting of $n$-capture elements.

\begin{tabular}{|c|c|c|c|c|}
\hline Element & $\begin{array}{c}a \\
{[\mathrm{dex}]}\end{array}$ & $\begin{array}{c}b \\
{\left[\operatorname{dex} \mathrm{Gyr}^{-1}\right]}\end{array}$ & $\begin{array}{c}\sigma_{[\mathrm{X} / \mathrm{Fe}]} \\
{[\mathrm{dex}]}\end{array}$ & $\chi_{\text {red }}^{2}$ \\
\hline $\mathrm{Y}$ & $0.141 \pm 0.010$ & $-0.033 \pm 0.002$ & 0.024 & 1.02 \\
\hline $\mathrm{Ba}$ & $0.207 \pm 0.027$ & $-0.035 \pm 0.005$ & 0.044 & 4.22 \\
\hline $\mathrm{La}$ & $0.161 \pm 0.026$ & $-0.027 \pm 0.005$ & 0.030 & 2.87 \\
\hline $\mathrm{Ce}$ & $0.192 \pm 0.037$ & $-0.030 \pm 0.007$ & 0.032 & 9.15 \\
\hline $\mathrm{Nd}$ & $0.160 \pm 0.020$ & $-0.024 \pm 0.004$ & 0.029 & 3.22 \\
\hline $\mathrm{Eu}$ & $0.092 \pm 0.009$ & $-0.013 \pm 0.002$ & 0.022 & 1.25 \\
\hline
\end{tabular}

enrichment of $n$-capture elements in the thin disk. However, this hypothesis assumes that all the $r$-process production occurs on sites of nucleosynthesis of very short timescales (e.g., SNe II) and that the contribution of the $r$-process is negligible in the latest stage of galactic evolution. This hypothesis is not necessarily true since colliding neutron stars can produce $r$-process elements (e.g., Wanajo et al. 2014), although we note that the timescales of these mergers are also short since a larger fraction $(\sim 70 \%)$ have a coalescence timescale $<1$ Gyr (Vangioni et al. 2016).

Since the AGB yields are highly dependent on the progenitor mass (Fishlock et al. 2014) and on metallicity (Busso et al. 2001; Karakas \& Lugaro 2016), we expect to observe non-linear $[\mathrm{X} / \mathrm{Fe}]$-age relations for the $s$-process elements resulting from the delayed pollution from low-mass AGB stars with respect to the early contribution from the intermediate-mass AGB stars. Unfortunately, the few abundance determinations for the $n$ capture elements in solar twins prevents us from estabilishing eventual non-linearities in the $[\mathrm{X} / \mathrm{Fe}]$ vs. age plots shown in Fig. 4. Better constraints in these relations would ensure powerful diagnostics of the AGB progenitor mass distribution.

The thick disk stars have [Y/Fe] ratios that are slightly higher than the ratios determined for most of the oldest thin disk stars. The change in slopes for the two different populations has already been noted by Battistini \& Bensby (2016) for a number of $n$-capture elements. They argue that the $[\mathrm{X} / \mathrm{Fe}]$ increase that

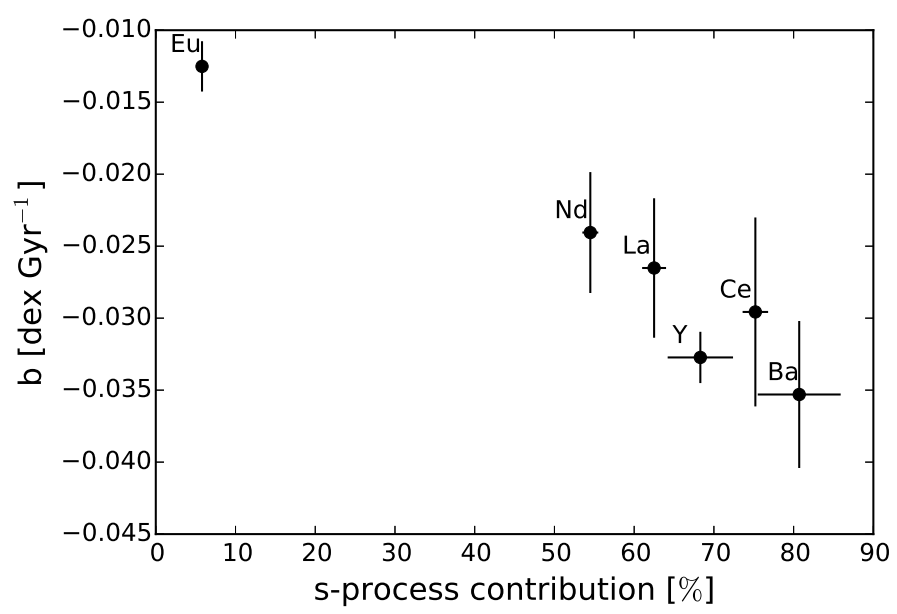

Fig. 5. Slopes of the $[\mathrm{X} / \mathrm{Fe}]-$ age relations found for the $n$-capture elements (i.e., the b parameters in Table 4) as a function of their solar $s$-process contribution percentages taken from Bisterzo et al. (2014).

they observed for $\mathrm{Sr}, \mathrm{Zr}, \mathrm{La}, \mathrm{Nd}, \mathrm{Sm}$, and $\mathrm{Eu}$ in stars older than $8 \mathrm{Gyr}$ is due to the production of these elements via SNe II, but it could also be due to massive stars (e.g., Hansen et al. 2014; Frischknecht et al. 2016).

\subsection{Chemical clocks}

The plot shown in Fig. 6 confirms that, as already found by N15 and Tucci Maia et al. (2016), the [Y/Mg] ratio is a sensitive indicator of age. A linear fit that includes both the thin and thick disk stars and that takes into account the errors in both the coordinates, results in the relation

$$
\left[\frac{\mathrm{Y}}{\mathrm{Mg}}\right]=0.176( \pm 0.011)-0.0410( \pm 0.0017) \times \text { Age }[\mathrm{Gyr}]
$$


L. Spina et al.: Nucleosynthetic history of elements in the Galactic disk
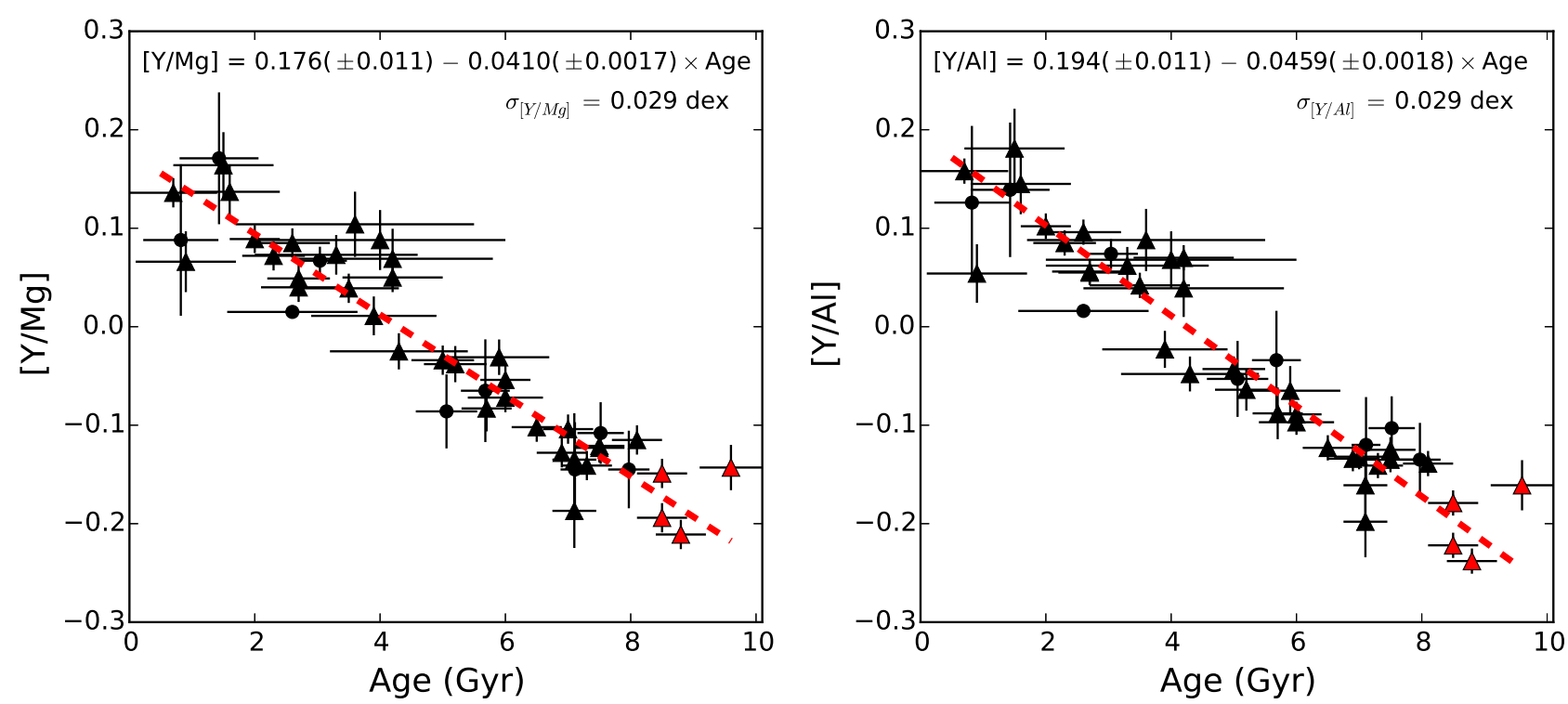

Fig. 6. $[\mathrm{Y} / \mathrm{Mg}]$ and $[\mathrm{Y} / \mathrm{Al}]$ as a function of age (symbols as in Fig. 1). The red dashed lines correspond to linear fits.

which is in excellent agreement with the relations provided by N15 and Tucci Maia et al. (2016). However, it is noteworthy that [Y/Al] also shows a very tight correlation with age and that, most importantly, this [Y/Al]-age dependence is steeper than that exhibited by $[\mathrm{Y} / \mathrm{Mg}]$ :

$\left[\frac{\mathrm{Y}}{\mathrm{Al}}\right]=0.194( \pm 0.011)-0.0459( \pm 0.0018) \times$ Age $[\mathrm{Gyr}]$.

The two relations have similar level of scatter (i.e., $\sigma_{[\mathrm{Y} / \mathrm{Mg}]}=$ 0.029 and $\sigma_{[\mathrm{Y} / \mathrm{Al}]}=0.029 \mathrm{dex}$ ), thus both the $[\mathrm{Y} / \mathrm{Mg}]$ and [Y/Al] ratios can serve as sensitive age indicators that are completely independent from the classical methods used for age determinations (e.g., isochrones, Li depletion, gyrochronology). We note that the slightly higher steepness of the [Y/Al]-age relation would provide better constraints on the stellar ages with respect to the $[\mathrm{Y} / \mathrm{Mg}]$ clock.

\section{Summary and conclusions}

Using high-quality UVES spectra of nine solar twins, we obtained precise estimates of stellar ages and chemical abundances. These determinations have been integrated with additional accurate age and abundance determinations from recent spectroscopy studies of 32 solar twins existing in the literature (Ramírez et al. 2011; Tucci Maia et al. 2014; Nissen 2015; Spina et al. 2016; Yana Galarza et al. 2016). So far, this represents the largest data set from which high-precision abundances of 24 species have been determined in order to outline the $[\mathrm{X} / \mathrm{Fe}]-$ age relations over a time interval of $\sim 10 \mathrm{Gyr}$.

Our main results can be summarized as follows:

- The thin and thick disk populations are clearly distinguishable by both the stellar ages and chemical composition (see Figs. 1 and 4). All the thick disk stars are older than $8 \mathrm{Gyr}$ and are enhanced in the $\alpha$-elements with respect to the thin disk population.

- Among the thin disk stars, tight correlations exist between the $[\mathrm{X} / \mathrm{Fe}]$ ratios and the stellar ages for many elements that we analyzed (see Fig. 1). We fitted the [X/Fe]-age relation of the light elements (i.e., from $\mathrm{C}$ to $\mathrm{Zn}$ ) using three different models based on a straight line, a hyperbolic function, and a two-segmented line function. Through a statistical comparison of the results, we demonstrated that most of these species present non-linear or non-monotonic $[\mathrm{X} / \mathrm{Fe}]-$ age relations. We showed that the hyperbolic model is the most appropriate in fitting the $[\mathrm{X} / \mathrm{Fe}]-$ ages distribution of most of the light elements. This hyperbolic fit provides age estimates of the knees of the $[\mathrm{X} / \mathrm{Fe}]$-age relations. Nevertheless, the $[\mathrm{X} / \mathrm{Fe}]$ ratios of $\mathrm{C}, \mathrm{O}, \mathrm{Al}$, and $\mathrm{Ti}$ show a more linear dependence to age.

- A relation connects the age at the knees of each species and their logarithmic ratios between the SN II and SN Ia yields (see Fig. 2). This relationship reflects the different histories of nucleosynthesis that each element underwent in the thin disk.

- The stars with ages $\sim 6-8$ Gyr are the most enriched in $\alpha$ elements among the thin disk population (Fig. 1). This early enrichment in the species mostly produced by SNe II could be due to an initial and intensive star formation burst that gave origin to the thin disk. For stars younger than 6-7 Gyr, the $[\mathrm{X} / \mathrm{Fe}]$ ratios of the $\alpha$-elements declines linearly with time. This decrement reflects the contribution of SNe Ia to the chemical evolution of the disk, which started to be nonnegligible $\sim 1$ Gyr after the initial stellar formation burst.

- Like the $\alpha$-elements, Na is mainly produced by SNe II; however, it shows an early $[\mathrm{X} / \mathrm{Fe}]$ increase that is somewhat more protracted in time with respect to the $\alpha$-elements (Fig. 1). This peculiarity could be due to the high metallicity dependency of the Na yields from SNe II.

- The species that are synthesized by SNe Ia present an early $[\mathrm{X} / \mathrm{Fe}]$ increment that is extended in time (see Fig. 1) and that could be the effect of the $\sim 1$ Gyr delay in the contribution from $\mathrm{SNe}$ Ia from the star formation burst. This early $[\mathrm{X} / \mathrm{Fe}]$ increase ends at $4-6 \mathrm{Gyr}$ and is followed by a $[\mathrm{X} / \mathrm{Fe}]$ decrease towards younger stars. The evolution of the ironpeak elements may be interpreted by invoking multiple types of $\mathrm{SNe} \mathrm{Ia}$, which are triggered by different progenitors and operate with different timescales.

- The elements $\mathrm{Ca}$ and $\mathrm{Cr}$ are characterized by a $[\mathrm{X} / \mathrm{Fe}]$ increase with time for the youngest stars (Fig. 1). These trends 
are also difficult to interpret based on the classical yields of $\mathrm{SNe}$ II and Ia. For instance, it is striking that $\mathrm{Ca}$ and the $\alpha$ elements show opposite behaviors.

- The $[\mathrm{X} / \mathrm{Fe}]$ ratios of the $n$-capture elements increase with time towards the youngest stars (see Fig. 4). The slopes of the $[\mathrm{X} / \mathrm{Fe}]-$ age relations are related to the contribution percentages to each element from the $s$-process (see Fig. 5): the elements that are mainly produced by the $s$-process are those with steeper slopes. This suggests that the enrichment in $n$-capture elements of the thin disk is mainly operated by AGB stars.

- The [Y/Mg] and [Y/Al] ratios are both powerful age indicators (see Fig. 6). However, of the two, the [Y/Al] ratio may be the better stellar clock since its dependence on age is slightly steeper than that found for $[\mathrm{Y} / \mathrm{Mg}]$.

Knowledge of the $[\mathrm{X} / \mathrm{Fe}]$-age relations is a gold mine from which we can extract a great understanding about the processes that governed the formation and evolution of the Milky Way (Haywood et al. 2013, 2016; Snaith et al. 2015): the nature of the star formation history, the SNe rates, the stellar yields, and the variety of the SNe progenitors, etc. Studies like Nissen (2015) and this work pave the way to a reverse engineering effort, which is both timely and necessary to reconstruct - through chemical evolution models (e.g., Chiappini et al. 1997; Matteucci et al. 2009; Romano et al. 2010; Kobayashi \& Nakasato 2011; Minchev et al. 2014; Snaith et al. 2015; Andrews et al. 2016) the nucleosynthetic history of the elements in the Galactic disk.

Acknowledgements. The authors are grateful to the anonymous referee, who made several important suggestions that have resulted in an improved body of work. We also thank Ashley Ruiter for comments on Type Ia supernovae. L.S. and J.M. acknowledge the support from FAPESP (2014/15706-9 and 2012/24392-2). M.A. and D.Y. acknowledge support from the Australian Research Council (grants FL110100012, DP120100991 and FT140100554).

\section{References}

Adibekyan, V. Z., Santos, N. C., Sousa, S. G., \& Israelian, G. 2011, A\&A, 535, L11

Amarsi, A. M., Asplund, M., Collet, R., \& Leenaarts, J. 2016, MNRAS, 455, 3735

Andrews, B. H., Weinberg, D. H., Schönrich, R., \& Johnson, J. A. 2016, ApJ, submitted [arXiv: 1604.08613]

Argast, D., Samland, M., Thielemann, F.-K., \& Qian, Y.-Z. 2004, A\&A, 416, 997

Arlandini, C., Käppeler, F., Wisshak, K., et al. 1999, ApJ, 525, 886

Battistini, C., \& Bensby, T. 2016, A\&A, 586, A49

Bedell, M., Meléndez, J., Bean, J. L., et al. 2014, ApJ, 795, 23

Bensby, T., Zenn, A. R., Oey, M. S., \& Feltzing, S. 2007, ApJ, 663, L13

Bensby, T., Feltzing, S., \& Oey, M. S. 2014, A\&A, 562, A71

Bergemann, M., Lind, K., Collet, R., Magic, Z., \& Asplund, M. 2012, MNRAS, 427, 27

Biazzo, K., Gratton, R., Desidera, S., et al. 2015, A\&A, 583, A135

Bisterzo, S., Travaglio, C., Gallino, R., Wiescher, M., \& Käppeler, F. 2014, ApJ 787,10

Boggs, P. T., \& Rogers, J. E. 1990, Contemporary Mathematics, 112, 183

Bonaparte, I., Matteucci, F., Recchi, S., et al. 2013, MNRAS, 435, 2460

Bours, M. C. P., Toonen, S., \& Nelemans, G. 2013, A\&A, 552, A24

Busso, M., Gallino, R., \& Wasserburg, G. J. 1999, ARA\&A, 37, 239

Busso, M., Marengo, M., Travaglio, C., Corcione, L., \& Silvestro, G. 2001, Mem. Soc. Astron. It., 72, 309

Castelli, F., \& Kurucz, R. L. 2004, ArXiv e-prints [arXiv: astro-ph/0405087]

Cayrel, R., \& Cayrel de Strobel, G. 1966, ARA\&A, 4, 1

Chamulak, D. A., Meakin, C. A., Seitenzahl, I. R., \& Truran, J. W. 2012, ApJ, 744, 27

Chiappini, C., Matteucci, F., \& Gratton, R. 1997, ApJ, 477, 765

Claeys, J. S. W., Pols, O. R., Izzard, R. G., Vink, J., \& Verbunt, F. W. M. 2014 , A\&A, 563, A83

Cowan, J. J., \& Sneden, C. 2004, Origin and Evolution of the Elements, 27

De, S., Timmes, F. X., Brown, E. F., et al. 2014, ApJ, 787, 149

D’Orazi, V., Magrini, L., Randich, S., et al. 2009, ApJ, 693, L31
Edvardsson, B., Andersen, J., Gustafsson, B., et al. 1993, A\&A, 275, 101

Ellis, R. S., Sullivan, M., Nugent, P. E., et al. 2008, ApJ, 674, 51

Epstein, C. R., Johnson, J. A., Dong, S., et al. 2010, ApJ, 709, 447

Fabbian, D., Asplund, M., Barklem, P. S., Carlsson, M., \& Kiselman, D. 2009, A\&A, 500, 1221

Fisher, R. 1925, Statistical Methods For Research Workers (Edinburgh: Oliver \& Boyd, UK)

Fishlock, C. K., Karakas, A. I., Lugaro, M., \& Yong, D. 2014, ApJ, 797, 44 Foley, R. J., Challis, P. J., Chornock, R., et al. 2013, ApJ, 767, 57

Frischknecht, U., Hirschi, R., Pignatari, M., et al. 2016, MNRAS, 456, 1803

Gibson, B. K., Fenner, Y., Renda, A., Kawata, D., \& Lee, H.-C. 2003, PASA, 20, 401

Gilmore, G., Wyse, R. F. G., \& Kuijken, K. 1989, ARA\&A, 27, 555

Graham, M. L., Nugent, P. E., Sullivan, M., et al. 2015, MNRAS, 454, 1948

Han, Z., \& Podsiadlowski, P. 2004, MNRAS, 350, 1301

Hansen, C. J., Montes, F., \& Arcones, A. 2014, ApJ, 797, 123

Haywood, M., Di Matteo, P., Lehnert, M. D., Katz, D., \& Gómez, A. 2013, A\&A, 560, A109

Haywood, M., Di Matteo, P., Snaith, O., \& Lehnert, M. D. 2015, A\&A, 579, A5

Haywood, M., Lehnert, M. D., Di Matteo, P., et al. 2016, A\&A, 589, A66

Hillebrandt, W., Kromer, M., Röpke, F. K., \& Ruiter, A. J. 2013, Frontiers of Physics, 8, 116

Howell, D. A., Sullivan, M., Brown, E. F., et al. 2009, ApJ, 691, 661

Iben, Jr., I., \& Tutukov, A. V. 1984, ApJ, 284, 719

Jackson, A. P., Calder, A. C., Townsley, D. M., et al. 2010, ApJ, 720, 99

Jiménez, N., Tissera, P. B., \& Matteucci, F. 2015, ApJ, 810, 137

Karakas, A. I. 2010, MNRAS, 403, 1413

Karakas, A. I., \& Lattanzio, J. C. 2014, PASA, 31, e030

Karakas, A. I., \& Lugaro, M. 2016, ApJ, 825, 26

Kelly, P. L., Hicken, M., Burke, D. L., Mandel, K. S., \& Kirshner, R. P. 2010, ApJ, 715, 743

Kim, Y.-C., Demarque, P., Yi, S. K., \& Alexander, D. R. 2002, ApJS, 143, 499

Kobayashi, C., \& Nakasato, N. 2011, ApJ, 729, 16

Kobayashi, C., Tsujimoto, T., Nomoto, K., Hachisu, I., \& Kato, M. 1998, ApJ, 503, L155

Kobayashi, C., Umeda, H., Nomoto, K., Tominaga, N., \& Ohkubo, T. 2006, ApJ, 653,1145

Korobkin, O., Rosswog, S., Arcones, A., \& Winteler, C. 2012, MNRAS, 426, 1940

Kratz, K.-L., Farouqi, K., Pfeiffer, B., et al. 2007, ApJ, 662, 39

Langer, N., Deutschmann, A., Wellstein, S., \& Höflich, P. 2000, A\&A, 362, 1046

Lemasle, B., de Boer, T. J. L., Hill, V., et al. 2014, A\&A, 572, A88

Letarte, B., Hill, V., Tolstoy, E., et al. 2010, A\&A, 523, A17

Lind, K., Asplund, M., Barklem, P. S., \& Belyaev, A. K. 2011, A\&A, 528, A103

Magrini, L., Sestito, P., Randich, S., \& Galli, D. 2009, A\&A, 494, 95

Maiorca, E., Randich, S., Busso, M., Magrini, L., \& Palmerini, S. 2011, ApJ, 736,120

Mannucci, F., Della Valle, M., \& Panagia, N. 2006, MNRAS, 370, 773

Maoz, D., Mannucci, F., \& Nelemans, G. 2014, ARA\&A, 52, 107

Matteucci, F. 2014, The Origin of the Galaxy and Local Group, Saas-Fee Advanced Course (Berlin Heidelberg: Springer-Verlag), 37, 145

Matteucci, F., \& Greggio, L. 1986, A\&A, 154, 279

Matteucci, F., \& Recchi, S. 2001, ApJ, 558, 351

Matteucci, F., Spitoni, E., Recchi, S., \& Valiante, R. 2009, A\&A, 501, 531

Meléndez, J., Bergemann, M., Cohen, J. G., et al. 2012, A\&A, 543, A29

Meléndez, J., Ramírez, I., Karakas, A. I., et al. 2014, ApJ, 791, 14

Meléndez, J., Bedell, M., Bean, J. L., et al. 2016, A\&A, accepted

Mennekens, N., Vanbeveren, D., De Greve, J. P., \& De Donder, E. 2010, A\&A, 515, A89

Miles, B. J., van Rossum, D. R., Townsley, D. M., et al. 2016, ApJ, 824, 59

Minchev, I., Chiappini, C., \& Martig, M. 2014, A\&A, 572, A92

Monroe, T. R., Meléndez, J., Ramírez, I., et al. 2013, ApJ, 774, L32

Nissen, P. E. 2015, A\&A, 579, A52

Nomoto, K., Hashimoto, M., Tsujimoto, T., et al. 1997a, Nucl. Phys. A, 616, 79

Nomoto, K., Iwamoto, K., Nakasato, N., et al. 1997b, Nucl. Phys. A, 621, 467

Pakmor, R., Kromer, M., Röpke, F. K., et al. 2010, Nature, 463, 61

Pearson, K. 1900, Philosophical Magazine, 5, 157

Perets, H. B., Gal-Yam, A., Mazzali, P. A., et al. 2010, Nature, 465, 322

Pipino, A., \& Matteucci, F. 2009, in The Ages of Stars, eds. E. E. Mamajek, D. R. Soderblom, \& R. F. G. Wyse, IAU Symp., 258, 39

Ramírez, I., Allende Prieto, C., \& Lambert, D. L. 2007, A\&A, 465, 271

Ramírez, I., Meléndez, J., Cornejo, D., Roederer, I. U., \& Fish, J. R. 2011, ApJ, 740,76

Ramírez, I., Fish, J. R., Lambert, D. L., \& Allende Prieto, C. 2012, ApJ, 756, 46 Ramírez, I., Allende Prieto, C., \& Lambert, D. L. 2013, ApJ, 764, 78

Ramírez, I., Meléndez, J., \& Asplund, M. 2014a, A\&A, 561, A7

Ramírez, I., Meléndez, J., Bean, J., et al. 2014b, A\&A, 572, A48

Recio-Blanco, A., de Laverny, P., Kordopatis, G., et al. 2014, A\&A, 567, A5 
Reddy, B. E., Lambert, D. L., \& Allende Prieto, C. 2006, MNRAS, 367, 1329 Romano, D., Karakas, A. I., Tosi, M., \& Matteucci, F. 2010, A\&A, 522, A32 Ruiter, A. J., Belczynski, K., \& Fryer, C. 2009, ApJ, 699, 2026

Ruiter, A. J., Belczynski, K., Sim, S. A., Seitenzahl, I. R., \& Kwiatkowski, D. 2014, MNRAS, 440, L101

Seitenzahl, I. R., Cescutti, G., Röpke, F. K., Ruiter, A. J., \& Pakmor, R. 2013, A\&A, 559, L5

Snaith, O. N., Haywood, M., Di Matteo, P., et al. 2014, ApJ, 781, L31

Snaith, O., Haywood, M., Di Matteo, P., et al. 2015, A\&A, 578, A87

Sneden, C. 1973, ApJ, 184, 839

Sneden, C., Cowan, J. J., \& Gallino, R. 2008, ARA\&A, 46, 241

Soubiran, C., Jasniewicz, G., Chemin, L., et al. 2013, A\&A, 552, A64

Spina, L., Meléndez, J., \& Ramírez, I. 2016, A\&A, 585, A152

Stritzinger, M., Leibundgut, B., Walch, S., \& Contardo, G. 2006, A\&A, 450, 241

Surman, R., McLaughlin, G. C., Ruffert, M., Janka, H.-T., \& Hix, W. R. 2008, ApJ, 679, L117

Thielemann, F.-K., Hashimoto, M.-A., \& Nomoto, K. 1990, ApJ, 349, 222

Thielemann, F.-K., Arcones, A., Käppeli, R., et al. 2011, Prog. Part. Nucl. Phys., 66,346

Timmes, F. X., Woosley, S. E., \& Weaver, T. A. 1995, ApJS, 98, 617

Timmes, F. X., Brown, E. F., \& Truran, J. W. 2003, ApJ, 590, L83

Tinsley, B. M. 1979, ApJ, 229, 1046

Travaglio, C., Hillebrandt, W., \& Reinecke, M. 2005, A\&A, 443, 1007
Travaglio, C., Gallino, R., Rauscher, T., Röpke, F. K., \& Hillebrandt, W. 2015, ApJ, 799, 54

Tsujimoto, T., \& Shigeyama, T. 2012, ApJ, 760, L38

Tucci Maia, M., Meléndez, J., \& Ramírez, I. 2014, ApJ, 790, L25

Tucci Maia, M., Meléndez, J., Castro, M., et al. 2015, A\&A, 576, L10

Tucci Maia, M., Ramírez, I., Meléndez, J., et al. 2016, A\&A, 590, A32

van Kerkwijk, M. H., Chang, P., \& Justham, S. 2010, ApJ, 722, L157

van Leeuwen, F. 2007, A\&A, 474, 653

Vangioni, E., Goriely, S., Daigne, F., François, P., \& Belczynski, K. 2016, MNRAS, 455, 17

Venn, K. A., Irwin, M., Shetrone, M. D., et al. 2004, AJ, 128, 1177

Vincenzo, F., Matteucci, F., Belfiore, F., \& Maiolino, R. 2016, MNRAS, 455, 4183

Wanajo, S., Sekiguchi, Y., Nishimura, N., et al. 2014, ApJ, 789, L39

Wang, B., Justham, S., \& Han, Z. 2013, A\&A, 559, A94

Webbink, R. F. 1984, ApJ, 277, 355

Winteler, C., Käppeli, R., Perego, A., et al. 2012, ApJ, 750, L22

Yana Galarza, J., Meléndez, J., Ramírez, I., et al. 2016, A\&A, 589, A17

Yi, S., Demarque, P., Kim, Y.-C., et al. 2001, ApJS, 136, 417

Yoshii, Y., Tsujimoto, T., \& Nomoto, K. 1996, ApJ, 462, 266

Yungelson, L. R. 2010, Astron. Lett., 36, 780

Yungelson, L. R., \& Livio, M. 2000, ApJ, 528, 108 


\section{Appendix A: Additional table}

Table A.1. $[\mathrm{X} / \mathrm{Fe}]$ ratios from our analysis.

\begin{tabular}{|c|c|c|c|c|c|c|}
\hline Star & $\mathrm{C}$ & $\mathrm{O}$ & $\mathrm{Na}$ & $\mathrm{Mg}$ & $\mathrm{Al}$ & $\mathrm{Si}$ \\
\hline $18 \mathrm{Sco}$ & $.063 \pm 0.015(5)$ & $0.050 \pm 0.009(3)$ & $0.031 \pm 0.007$ & $-0.014 \pm 0.012$ & $-0.021 \pm 0.013$ & $-0.007 \pm 0.0$ \\
\hline HIP 56 & $011 \pm 0.015$ (5) & $0.005 \pm 0.011(3)$ & $-0.004 \pm 0.011$ & & & 2) \\
\hline 2 & & & & & & \\
\hline HD 2 & $-0.106 \pm 0$ & 0.008 & -0.0 & -0.0 & 6) & -0. \\
\hline HD 2 & 4) & 0.0 & -0.0 & -0 . & & -0 . \\
\hline HIP 3 & \pm 0 & -0.01 & -0.0 & ) & & 3) \\
\hline 5 & 4) & -0.0 & -0. & & & \\
\hline HIP 7 & -0.0 & 0.00 & -0.0 & ) & 0 . & 3) \\
\hline HIP 8 & \pm 0.0 & $=0.0$ & \pm 0 & \pm 0. & & 13) \\
\hline Star & $\mathrm{S}$ & $\mathrm{K}$ & $\mathrm{Ca}$ & $\mathrm{Sc}$ & $\mathrm{Ti}$ & $\mathrm{V}$ \\
\hline $18 \mathrm{Sco}$ & $-0.038 \pm 0.0$ & \pm 0.0 & $0.002 \pm 0.0$ & $.006 \pm 0.0$ & $-0.002 \pm 0.010$ & $-0.013 \pm 0$ \\
\hline HIP 5 & -0.01 & \pm 0.0 & 0.00 & 2) & & \\
\hline HIP 1 & $-0.001 \pm 0.02$ & $0.010 \pm 0.0$ & 0.01 & \pm 0 & $0.012 \pm 0.00$ & $-0.021 \pm 0$ \\
\hline $\mathrm{HD} 2$ & $-0.026 \pm 0.0$ & & 0.02 & & & \\
\hline $\mathrm{HD} 2$ & -0.0 & 0.03 & 0.03 & \pm 0. & 0.01 & -0 \\
\hline HIP 3 & 6) & -0. & & & & \\
\hline HIP & \pm 0 & $=0.0$ & -0 & $9)$ & 1) & -0 \\
\hline HIP & & 1) & & & & -0 \\
\hline HIP 8 & \pm 0.0 & $.038 \pm 0.0$ & \pm 0.0 & \pm 0.0 & -0 & $1 \pm 0$. \\
\hline Star & $\mathrm{Cr}$ & Mn & Co & $\mathrm{Ni}$ & $\mathrm{Cu}$ & $\mathrm{Zn}$ \\
\hline $18 \mathrm{Sc}$ & $07+00$ & +0 & \pm 0 & 9) & t) & 3) \\
\hline HIP : & \pm 0. & $=0$. & 0.0 & & -0 & $0 .($ \\
\hline HIP 1 & \pm 0 & - & 3) & 7) & & 2) \\
\hline HD 2 & 6) & -0. & -0 . & -0 & -0 & -0 \\
\hline HD 2 & 6) & -0.0 & -0 & 9) & -0 & -0 \\
\hline HIP 3 & $-0.003 \pm 0$ & -0.0 & \pm 0 & -0. & $-0 .($ & -0 . \\
\hline HIP 7 & $.008 \pm 0.0$ & -0.0 & +0 & -0 & +0 & (2) \\
\hline HIP 7 & $.005 \pm 0.009(16)$ & $-0.004 \pm 0.0$ & $.003 \pm 0$ & -0.0 & 0.0 & $0.016 \pm 0.0$ \\
\hline HIP 8 & $004 \pm 0.0$ & $-0.005 \pm 0$. & $21 \pm 0.0$ & $0.004 \pm 0.0$ & $0.030 \pm 0.024$ & $0.021 \pm 0.00$ \\
\hline Star & $\mathrm{Y}$ & $\mathrm{Ba}$ & $\mathrm{La}$ & $\mathrm{Ce}$ & $\mathrm{Nd}$ & $\mathrm{Eu}$ \\
\hline $18 \mathrm{Sco}$ & $3 \pm 0$. & \pm 0. & \pm 0. & \pm 0.0 & \pm 0 . & \pm 0 \\
\hline HIP 5 & $00 \pm 0.00$ & $0.002 \pm 0.0$ & 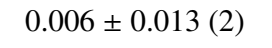 & -8 & & \\
\hline HIP 1 & $-0.120 \pm 0.0$ & $-0.014 \pm 0.0$ & $-0.029 \pm 0.0$ & $-0.018 \pm 0.00$ & & $3 \pm 0$ \\
\hline $\mathrm{HD} 2$ & $0.088 \pm 0$. & $0.192 \pm 0.0$ & \pm 0. & 0.1 & \pm 0 & (1) \\
\hline & 0 & 0 & & & & (1) \\
\hline HIP 3 & $-0.035 \pm 0.044(5)$ & $0.037 \pm 0.016(3)$ & $0.010 \pm 0$. & & $0.086 \pm 0.0$ & $0.032 \pm 0.0$ \\
\hline & $-0.124 \pm 0$ & $-0.020 \pm 0$ & $-0.049 \pm 0$ & -0 & $36(2)$ & $-0.008 \pm 0$ \\
\hline HIP 77883 & $-0.097 \pm 0.030(5)$ & $-0.016 \pm 0.014$ & $-0.011 \pm 0.009$ & $-0.042 \pm 0.007(1)$ & $-0.022 \pm 0.031$ & $-0.002 \pm 0.008$ \\
\hline HIP 89650 & $-0.050 \pm 0.033(5)$ & $-0.004 \pm 0.011(3)$ & $0.019 \pm 0.015(1)$ & $0.043 \pm 0.009(1)$ & $0.012 \pm 0.054(2)$ & $0.021 \pm 0.009(1)$ \\
\hline
\end{tabular}

Notes. The number of lines that have been detected for each element are in parentheses. 